\title{
Epicyclic Oscillations of Fluid Bodies: Newtonian Non-Slender Torus
}

\author{
Omer M. Blaes ${ }^{1}$ \\ blaes@physics.ucsb.edu \\ Eva Šrámková \\ sram_eva@centrum.cz \\ Marek A. Abramowicz $3,4,2$ \\ marek@fy . chalmers.se \\ Włodek Kluźniak ${ }^{4,5}$ \\ wlodek@camk.edu.pl \\ and \\ Ulf Torkelsson ${ }^{3}$ \\ torkel@fy.chalmers.se
}

\begin{abstract}
We study epicyclic oscillations of fluid tori around black holes (in the Paczyński-Wiita potential), and derive exact analytic expressions for their radial and vertical eigenfrequencies $\nu_{r}$ and $\nu_{z}$, to second order accuracy in the width of the torus. We prove that pressure effects make the eigenfrequencies smaller than those for free particles. However, the particular ratio $\nu_{z} / \nu_{r}=3 / 2$, that is important for the theory of high frequency QPOs, occurs when the fluid tori
\end{abstract}

\footnotetext{
${ }^{1}$ Department of Physics, University of California, Santa Barbara, CA 93106

${ }^{2}$ Institute of Physics, Silesian University in Opava, Bezručovo nám. 13, 74601 Opava, Czech Republic

${ }^{3}$ Department of Physics, Göteborg University, S-412 96 Göteborg, Sweden

${ }^{4}$ Copernicus Astronomical Centre, Bartycka 18, PL-00-716 Warszawa, Poland

${ }^{5}$ Institute of Astronomy, Zielona Góra University, Wieża Braniborska, Lubuska 2, PL-65-265 Zielona Góra, Poland
} 
epicyclic frequencies $\nu_{r}, \nu_{z}$ are about $15 \%$ higher than the ones corresponding to free particles. Our results therefore suggest that previous estimates of black hole spins from QPOs have produced values that are too high.

Subject headings: accretion, accretion disks — black hole physics — hydrodynamics - X-rays: binaries

\section{Introduction}

The Fourier power density spectra of X-ray variability in Galactic black hole X-ray binaries often reveal pairs of high frequency QPOs (e.g., Strohmaver 2001; Remillard \& McClintock 2006). Kluźniak \& Abramowicz (2001a,b) suggested that these high frequency QPOs are caused by a non-linear resonance between two global modes of oscillations in an accretion flow in strong gravity (here we denote these modes by $\delta r, \delta z$ ), and pointed out that the observed frequencies are in a commensurate (3:2) ratio. This suggestion was developed by them and collaborators into the "QPO resonance model". The model uses the theory of small non-linear oscillations (e.g., Nayfeh \& Mook 1979), and attempts to explain many observational properties of QPOs in X-ray binaries by deriving them directly from the differential equations that describe two weakly coupled, non-linear oscillators (for more information, see the collection of reviews in Abramowicz 2005b),

$$
\begin{aligned}
\delta \ddot{r}+\left(\omega_{r}\right)^{2} \delta r & =\mathcal{X}_{r}(\delta r, \delta \dot{r}, \delta z, \delta \dot{z}), \\
\delta \ddot{z}+\left(\omega_{z}\right)^{2} \delta z & =\mathcal{X}_{z}(\delta r, \delta \dot{r}, \delta z, \delta \dot{z}) .
\end{aligned}
$$

The resonance model does not address, however, the actual accretion flow structure or the specific modes of oscillation, information upon which the detailed form of equations (1) depend.

One possibility is that radial pressure gradients set up fluid tori in the accretion flow which can support discrete, trapped hydrodynamic modes. That oscillations of such tori might be an interesting model for QPOs was first recognized by Rezzolla and his collaborators (Zanotti. Rezzolla \& Font 2003; Rezzolla et al. 2003; see also Lee, Abramowicz \& Kluźniak 2004, Rubio-Herrera \& Lee 2005, and Blaes. Arras \& Fragile 2006). It is not yet clear whether such tori provide a realistic model for the accretion flow in the steep power law state (Remillard \& McClintock 2006), where high frequency QPOs are observed. Nor is it clear whether their hydrodynamic modes of oscillation can exist in the presence of magnetorotational (MRI) turbulence. Nevertheless, pressure supported "inner tori" do appear to be an ubiquitous flow feature of nonradiative global simulations of MRI turbulence in 
accretion flows (Hawley \& Balbus 2002; De Villiers. Hawlev \& Krolik 2003). An example of such an inner torus is shown in Figure 1 (Machida et al. 2006).

If torus-like structures do exist in the steep power law state, global epicyclic oscillations of these tori are almost certainly the most robust modes, as their existence is derived from the properties of the external spacetime, not the internal properties of the torus (Abramowicz et al. 2006). While the existence of these modes is independent of the properties of the torus, their actual frequencies and eigenfunctions are not. It is this issue which we wish to address in the present paper: how the frequencies of epicyclic modes of thick (nonslender) fluid tori differ from the epicyclic frequencies of test particles. As we shall discuss later in this paper, this question is of direct relevance for an accurate estimate of the black hole spin from the measured QPO frequencies. In order to answer this question, we calculate analytically eigenfrequencies and eigenfunctions of the epicyclic modes of nonslender tori up to the second order in the torus thickness.

At first sight, vertical and radial epicyclic modes would seem to be a terrible choice for a resonance, as test particle orbits in Kerr spacetime are fully separable and there is therefore no nonlinear coupling between these motions. However, tori behave like test particles only when they are very slender. Kluźniak \& Abramowicz (2002) recognized that for nonslender tori the frequencies of the epicyclic modes would be modified by pressure. They derived an approximate formula for the epicyclic frequencies, radial $\omega_{r}$ and vertical $\omega_{z}$, of fluid tori,

$$
\left(\omega_{r}\right)^{2}=\left(\omega_{r}^{0}\right)^{2}-A_{r} c_{s 0}^{2} \text { and }\left(\omega_{z}\right)^{2}=\left(\omega_{z}^{0}\right)^{2}-A_{z} c_{s 0}^{2} .
$$

Here $\omega_{r}^{0}$ and $\omega_{z}^{0}$ are the radial and vertical epicyclic frequencies for particles, $c_{s 0}$ is the sound speed at the torus center, and the coefficients $A_{r}$ and $A_{z}$ are (not exactly specified) functions of the equation of state and the background gravitational potential. Numerical work by Rubio-Herrera \& Lee (2005) revealed that $A_{r}>0$ and $A_{z}>0$. In this paper we analytically calculate explicit forms of $A_{r}$ and $A_{z}$. Another motivation for the present work is that it is a necessary step toward deriving an explicit form of equations (1). In the slender torus limit, there is no nonlinear coupling of the epicyclic modes, but the pressure corrections of equations (2) may give rise to nontrivial couplings.

For simplicity we model general relativistic effects throughout this paper with the pseudo-Newtonian potential of Paczyński \& Wiita (1980). The mathematics of nonslender tori is complicated, and a Newtonian calculation is a useful first step before attempting the calculation in full Kerr geometry. We will publish an extention of our results to the Kerr geometry separately, in O. Straub et al. (2007, in preparation). In any case, the exact analytic results here will be useful for oscillatory mode identification in numerically simulated Paczyński-Wiita flows, as already attempted by Bursa (2006) and M. Bursa \& M. Machida (2007, in preparation). 
This paper is organized as follows. In Section 2 we briefly review the equilibrium structure of tori, citing results that we will need later. In Section 3 we demonstrate that radial and vertical epicyclic modes exist for a completely general, baroclinic slender torus. In Section 4 we then restrict consideration to polytropic, constant specific angular momentum tori and derive the lowest order pressure corrections to the epicyclic mode frequencies (second order) and eigenfunctions (first order). We discuss our results and present our conclusions in Section 5 .

\section{Newtonian Slender Torus}

Consider an axially symmetric, inviscid rotating fluid body with toroidal topology in equilibrium in an external axially symmetric gravitational field. The flow is stationary and its velocity only has an azimuthal component, $\mathbf{v}=\Omega r \hat{\boldsymbol{\phi}}$. In the paper we use cylindrical coordinates $\{r, \phi, z\}$ for all calculations. The gravitational field is described by the potential $\Phi(r, z)$, which we assume possesses reflection symmetry: $\Phi(r, z)=\Phi(r,-z)$.

Dynamical equilibrium requires that

$$
\Omega^{2} \mathbf{r}=\frac{\nabla p}{\rho}+\nabla \Phi .
$$

Here $p$ is the pressure and $\rho$ is the density. Of particular interest is the circle where the pressure has zero gradient. We shall call this the equilibrium circle, as it corresponds to a balance between centrifugal and gravitational forces, as one can verify by substituting $\nabla p=0$ into equation (3). It follows from this equation that the circle lies in the equatorial plane at a distance $r_{0}$ where the rotational velocity $\Omega_{0}$ and the specific angular momentum $\ell_{0}$ of the flow have their test particle ("Keplerian") values $\Omega_{K}\left(r_{0}\right)$ and $\ell_{K}\left(r_{0}\right)$. Let us introduce the effective potential of a test particle with specific angular momentum $\ell_{0}, \mathcal{U} \equiv \Phi+\ell_{0}^{2} /\left(2 r^{2}\right)$. The equilibrium circle lies at its minimum and the Euler equation (3) can be rewritten as

$$
\frac{\ell^{2}-\ell_{0}^{2}}{r^{3}} \hat{\mathbf{r}}=\frac{\nabla p}{\rho}+\nabla \mathcal{U} .
$$

The full equilibrium structure can easily be derived from this equation in cases where the pressure can be expressed as a function of density alone (a barotropic or pseudo-barotropic equilibrium, e.g. Tassoul 1978). In this case, it is possible to find a potential $H$ such that $\nabla H=\nabla p / \rho$. The left-hand side of equation (4) can then be expressed as a gradient. Moreover, since this gradient has only a radial component, the corresponding potential is a function of $r$ only and also the angular momentum $\ell$ must be function of $r$ only - the specific angular momentum of the flow is constant on cylinders. 
We now assume that the equilibrium pressure and density obey a polytropic relation: $p \propto \rho^{1+1 / n}$. Let us define two potentials

$$
H=\int \frac{d p}{\rho}=(n+1) \frac{p}{\rho} \quad \text { and } \quad \Psi=-\int_{r_{0}}^{r} \frac{\ell^{2}\left(r^{\prime}\right)-\ell_{0}^{2}}{r^{\prime 3}} d r^{\prime} .
$$

Substituting these into equation (44), we obtain the Bernoulli equation in the form

$$
\mathcal{U}+\Psi+(n+1) \frac{p}{\rho}=\text { const. }
$$

The constant can be evaluated by considering the equation at the equilibrium point. Then we find

$$
\frac{p}{\rho}=\frac{p_{0}}{\rho_{0}}\left[1-\frac{1}{n c_{\mathrm{s} 0}^{2}}\left(\mathcal{U}-\mathcal{U}_{0}+\Psi\right)\right] \equiv \frac{p_{0}}{\rho_{0}} f(r, z),
$$

Here $c_{\mathrm{s} 0}^{2} \equiv(n+1) p_{0} /\left(n \rho_{0}\right)$, so that $c_{\mathrm{s} 0}$ is the adiabatic sound speed $c_{\mathrm{s}}$ evaluated at the equilibrium point if the barotropic equilibrium happens to also be isentropic. The pressure and density profiles are given by $\rho=\rho_{0} f^{n}$ and $p=p_{0} f^{n+1}$. Surfaces of constant pressure and density coincide with surfaces of constant $f$.

It is useful to examine the behavior of the function $f$ in the vicinity of the equilibrium point. For this purpose let us express the coordinates $r$ and $z$ as $r=(1+x) r_{0}$ and $z=y r_{0}$. The equilibrium point corresponds to $x=y=0$. For small $x$ and $y$ we have

$$
f=1-\frac{r_{0}^{2}}{2 n c_{\mathrm{s} 0}^{2}}\left[\left(\frac{\partial^{2} \mathcal{U}}{\partial r^{2}}\right)_{0} x^{2}+\left(\frac{\partial^{2} \mathcal{U}}{\partial z^{2}}\right)_{0} y^{2}-\frac{2 \ell_{0}}{r_{0}^{3}}\left(\frac{d \ell}{d r}\right)_{0} x^{2}\right] .
$$

The derivatives of the effective potential come from its expansion. The first derivatives are missing because the equilibrium point corresponds to a minimum of the effective potential. The mixed second derivatives vanish due to the reflection symmetry. The term containing the derivative of the specific angular momentum comes from the expansion of the potential $\Psi$. The second derivatives of the effective potential with respect to $r$ and $z$ give us radial and vertical epicyclic frequencies $\omega_{r}$ and $\omega_{z}$ in the equilibrium point. Let us express them as fractions of the orbital angular velocity $\Omega_{0}$ at the equilibrium circle, $\omega_{r}=\bar{\omega}_{r} \Omega_{0}, \omega_{z}=\bar{\omega}_{z} \Omega_{0}$. Then we find

$$
f=1-\frac{1}{\beta^{2}}\left\{\left[\bar{\omega}_{r}^{2}-\frac{2 r_{0}}{\ell_{0}}\left(\frac{d \ell}{d r}\right)_{0}\right] x^{2}+\bar{\omega}_{z}^{2} y^{2}\right\},
$$

where $\beta^{2} \equiv\left(2 n c_{\mathrm{s} 0}^{2}\right) /\left(r_{0}^{2} \Omega_{0}^{2}\right)$. The surfaces of constant pressure and density have elliptical or hyperbolic cross-sections depending on the sign of the square bracket. In fact, it is possible to express the radial epicyclic frequency using the gradient of the test particle ("Keplerian") specific angular momentum as $\bar{\omega}_{r}^{2}=\left(2 r_{0} / \ell_{0}\right)\left(d \ell_{\mathrm{K}} / d r\right)_{0}$. Therefore, when the specific angular momentum of the fluid increases more slowly than "Keplerian" (with increasing $r$ ), the 
equipressure surfaces have elliptical shape and the equilibrium point corresponds to the center of the torus with maximal pressure and density. When the increase is faster the equilibrium point is a cusp which corresponds to a saddle point in the pressure and density profiles.

Assume that the specific angular momentum distribution $\ell(r)$ is such that the equilibrium circle is at the center of the torus. The surface of the torus is the equipressure surface where $f=0$. Generally it can be far from the center - in the region where our approximation ( $\operatorname{small} x$ and $y$ ) is not valid. However, it is clear from equation (9) that our approximation is valid in the whole torus if $\beta$ is of the same order as $x$ and $y$, i.e., when the flow is highly supersonic. In this limit the torus becomes infinitesimally slender and its equipressure surfaces have elliptic shape. In the particular case of a constant specific angular momentum distribution, these ellipses have semiaxises in the ratio of the epicyclic frequencies. Moreover, when the external gravitational field is Newtonian, $\Phi \propto 1 /\left(r^{2}+z^{2}\right)^{1 / 2}$, these ellipses become circles. It follows that then the torus has a circular cross-section with radius $\beta r_{0}$.

\section{Epicyclic Modes of Baroclinic Slender Tori}

Keeping the assumption that the torus is made of an ideal fluid, we allow it to have arbitrary specific angular momentum $\ell(r, z)$ and entropy distributions. Surfaces of constant pressure and density need not coincide, and rotation need not be constant on cylinders. The equilibrium configuration must still satisfy equation (4), but now the right hand side of that equation cannot necessarily be expressed as a gradient, and we do not bother to attempt to solve this equation for a detailed equilibrium structure. However, we still assume that the torus equilibrium is slender.

For simplicity, we restrict consideration to axisymmetric perturbations of this equilibrium. The equations describing Eulerian perturbations are those of mass conservation, momentum conservation, and adiabatic flow:

$$
\begin{gathered}
\frac{\partial \delta \rho}{\partial t}+\frac{1}{r} \frac{\partial}{\partial r}\left(r \rho \delta v_{r}\right)+\frac{\partial}{\partial z}\left(\rho \delta v_{z}\right)=0 \\
\frac{\partial \delta v_{r}}{\partial t}-2 \Omega \delta v_{\phi}=\frac{-1}{\rho} \frac{\partial \delta p}{\partial r}+\frac{\delta \rho}{\rho^{2}} \frac{\partial p}{\partial r} \\
\frac{\partial \delta v_{\phi}}{\partial t}+\frac{1}{r} \delta \mathbf{v} \cdot \nabla \ell=0 \\
\frac{\partial \delta v_{z}}{\partial t}=\frac{-1}{\rho} \frac{\partial \delta p}{\partial z}+\frac{\delta \rho}{\rho^{2}} \frac{\partial p}{\partial z}
\end{gathered}
$$


and

$$
\frac{\partial \delta p}{\partial t}-c_{\mathrm{s}}^{2} \frac{\partial \delta \rho}{\partial t}+\delta \mathbf{v} \cdot\left(\nabla p-c_{\mathrm{s}}^{2} \nabla \rho\right)=0
$$

Now, for a slender torus, the derivatives of $r$ in the continuity equation become negligible, and we may write instead

$$
\frac{\partial \delta \rho}{\partial t}+\frac{\partial}{\partial r}\left(\rho \delta v_{r}\right)+\frac{\partial}{\partial z}\left(\rho \delta v_{z}\right)=0
$$

Hence from now on we may consider all vectors as two dimensional in $r$ and $z$, which may be treated as Cartesian coordinates as far as all vector operations are concerned. We consider possible modes in which $\delta v_{r}$ and $\delta v_{z}$ are spatially constant. Then after differentiating equations (11) and (13) with respect to time, and using equations (12), (14) and (15) to eliminate $\delta v_{\phi}, \delta p$, and $\delta \rho$, respectively, we obtain

$$
\frac{\partial^{2} \delta \mathbf{v}}{\partial t^{2}}+\hat{\mathbf{r}} \frac{2 \Omega}{r} \delta \mathbf{v} \cdot \nabla \ell=\delta \mathbf{v} \cdot \nabla\left(\frac{1}{\rho} \nabla p\right)
$$

For a slender torus, we may write

$\hat{\mathbf{r}} \frac{2 \Omega}{r} \delta \mathbf{v} \cdot \nabla \ell=\frac{\hat{\mathbf{r}}}{r^{3}} \delta \mathbf{v} \cdot \nabla\left(\ell^{2}-\ell_{0}^{2}\right) \simeq \frac{\hat{\mathbf{r}}}{r_{0}^{3}} \delta \mathbf{v} \cdot \nabla\left(\ell^{2}-\ell_{0}^{2}\right)=\hat{\mathbf{r}} \delta \mathbf{v} \cdot \boldsymbol{\nabla}\left(\frac{\ell^{2}-\ell_{0}^{2}}{r_{0}^{3}}\right) \simeq \delta \mathbf{v} \cdot \boldsymbol{\nabla}\left[\frac{\hat{\mathbf{r}}}{r^{3}}\left(\ell^{2}-\ell_{0}^{2}\right)\right]$.

Hence from the equilibrium condition (44), we finally obtain

$$
\frac{\partial^{2} \delta \mathbf{v}}{\partial t^{2}}=-\left.\delta \mathbf{v} \cdot \nabla(\nabla \mathcal{U})\right|_{0}
$$

The radial and vertical components of this equation give us our modes:

$$
\frac{\partial^{2} \delta v_{r}}{\partial t^{2}}=-\left(\frac{\partial^{2} \mathcal{U}}{\partial r^{2}}\right)_{0} \delta v_{r}=-\omega_{r}^{2} \delta v_{r}
$$

and

$$
\frac{\partial^{2} \delta v_{z}}{\partial t^{2}}=-\left(\frac{\partial^{2} \mathcal{U}}{\partial z^{2}}\right)_{0} \delta v_{z}=-\omega_{z}^{2} \delta v_{z}
$$

\section{Behavior of Epicylic Modes for Thick Tori}

We now turn to the behavior of the epicyclic modes for thicker tori. To keep things simple, we restrict consideration to polytropic tori with constant specific angular momentum. This is unlikely to be the most physically relevant case, particularly as existing global 
simulations of accretion flows with magnetorotational turbulence lead to near-Keplerian distributions of angular momentum (De Villiers, Hawley \& Krolik 2003; Machida et al. 2006; Matsumoto \& Machida 2007). However, the eigenvalue problem is self-adjoint for constant specific angular momentum, and the calculations are therefore far more straightforward. While there will presumably be quantitative differences for more general angular momentum distributions, there will still be pressure corrections to the modes which are likely to be comparable to those we calculate here. We discuss this issue further in Section 5.

Because the equilibrium tori are axisymmetric and stationary, we assume that all perturbations have azimuthal and time dependence $\propto \exp [i(m \phi-\omega t)]$. The periodicity of the solution in $\phi$ requires integer values of $m$.

Papaloizou \& Pringle (1984) have shown that the perturbation equations are simplest when expressed in terms of the variable

$$
W \equiv \frac{\delta p}{\rho(m \Omega-\omega)},
$$

where $\delta p$ is the Eulerian perturbation in pressure and $\Omega$ is the local angular velocity of fluid in the equilibrium torus. The Eulerian perturbation in fluid velocity is simply $\delta \mathbf{v}=i \nabla W$, so that $W$ is directly proportional to the perturbed velocity potential. In terms of $W$, the linear perturbations of the torus are governed by the equation

$$
\frac{1}{r} \frac{\partial}{\partial r}\left(r f^{n} \frac{\partial W}{\partial r}\right)+\frac{\partial}{\partial z}\left(f^{n} \frac{\partial W}{\partial z}\right)-\frac{m^{2}}{r^{2}} f^{n} W+\frac{2 n(\omega-m \Omega)^{2}}{\beta^{2} r_{0}^{2} \Omega_{0}^{2}} f^{n-1} W=0,
$$

where

$$
\beta^{2} \equiv \frac{2(n+1) p_{0}}{\rho_{0} r_{0}^{2} \Omega_{0}^{2}}
$$

is our slender torus perturbation parameter, and subscript zero refers to the pressure maximum of the torus. The equipotential function $f$ is given by

$$
f=1-\frac{2}{\beta^{2} r_{0}^{2} \Omega_{0}^{2}}\left(\mathcal{U}-\mathcal{U}_{0}\right)
$$

where

$$
\mathcal{U}=\Phi(r, z)+\frac{\ell_{0}^{2}}{2 r^{2}} .
$$

We assume the external gravitational potential $\Phi(r, z)$ is symmetric with respect to reflections about the equatorial plane, so all odd $z$-derivatives of $\mathcal{U}$ vanish in the equatorial plane.

The Papaloizou-Pringle equation may be written in abstract operator form as

$$
\hat{L} W=-2 n(\bar{\omega}-m \bar{\Omega})^{2} W .
$$


Here $\bar{\omega} \equiv \omega / \Omega_{0}$ and $\bar{\Omega} \equiv \Omega / \Omega_{0}$. Also,

$$
\hat{L} \equiv \beta^{2} r_{0}^{2}\left[f \frac{\partial^{2}}{\partial r^{2}}+\left(\frac{f}{r}+n \frac{\partial f}{\partial r}\right) \frac{\partial}{\partial r}+f \frac{\partial^{2}}{\partial z^{2}}+n \frac{\partial f}{\partial z} \frac{\partial}{\partial z}-\frac{m^{2} f}{r^{2}}\right]
$$

is a linear operator which is self-adjoint in the inner product

$$
<W_{1} \mid W_{2}>\equiv \frac{1}{\beta^{2} r_{0}^{3}} \int d r \int d z r f^{n-1} W_{1}^{\star} W_{2}
$$

where the double integral is taken over the torus cross-section defined by $f \geq 0$.

\subsection{Slender Torus Limit}

Change variables from $r$ and $z$ to

$$
\bar{x} \equiv \frac{r-r_{0}}{\beta r_{0}} \text { and } \quad \bar{y} \equiv \frac{z}{\beta r_{0}} \text {. }
$$

Then, in the slender torus limit $\beta \rightarrow 0$, the equipotential function becomes

$$
f^{(0)}=1-\bar{\omega}_{r}^{2} \bar{x}^{2}-\bar{\omega}_{z}^{2} \bar{y}^{2}
$$

where we use a superscript (0) to denote the slender torus limit. Here $\bar{\omega}_{r}$ and $\bar{\omega}_{z}$ are again the radial and vertical epicyclic frequencies at the pressure maximum, scaled with the angular velocity $\Omega_{0}$ :

$$
\bar{\omega}_{r}^{2}=\frac{1}{\Omega_{0}^{2}}\left(\frac{\partial^{2} \mathcal{U}}{\partial r^{2}}\right)_{0} \text { and } \quad \bar{\omega}_{z}^{2}=\frac{1}{\Omega_{0}^{2}}\left(\frac{\partial^{2} \mathcal{U}}{\partial z^{2}}\right)_{0} .
$$

The slender torus limit of the Papaloizou-Pringle equation is

$$
\hat{L}^{(0)} W^{(0)}+2 n \bar{\sigma}_{0}^{2} W^{(0)}=0
$$

where $\bar{\sigma}_{0} \equiv \bar{\omega}^{(0)}-m$ and

$$
\hat{L}^{(0)}=f^{(0)} \frac{\partial^{2}}{\partial \bar{x}^{2}}+n \frac{\partial f^{(0)}}{\partial \bar{x}} \frac{\partial}{\partial \bar{x}}+f^{(0)} \frac{\partial^{2}}{\partial \bar{y}^{2}}+n \frac{\partial f^{(0)}}{\partial \bar{y}} \frac{\partial}{\partial \bar{y}}
$$

is the slender torus limit of the linear operator $\hat{L}$. This is still a self-adjoint operator with respect to the inner product

$$
<W_{1} \mid W_{2}>=\int d \bar{x} \int d \bar{y}\left(f^{(0)}\right)^{n-1} W_{1}^{\star} W_{2},
$$


where the integrals are now taken over the domain where $f^{(0)} \geq 0$. Because of this fact, the eigenvalues $\bar{\sigma}_{0}^{2}$ are all real, and the corresponding eigenfunctions $W^{(0)}$ form a complete orthonormal set in which any regular function defined on the domain $f^{(0)} \geq 0$ may be expanded.

Blaes (1985) derived the full set of orthonormal modes explicitly for point mass potentials in which $\bar{\omega}_{r}=\bar{\omega}_{z}=1$. Blaes, Arras \& Fragile (2006) have derived the lowest order eigenfunctions and eigenfrequencies for the more general case considered here. The simplest modes are given in Table 1. We are interested in the behavior of the two epicyclic modes $(J=1$ and 2 in Table 1$)$ as the torus becomes thicker. In the slender torus limit,

$$
W_{1}^{(0)} \equiv a_{1} \bar{x} e^{i\left(m \phi-\omega_{1}^{(0)} t\right)} \quad \text { and } \quad W_{2}^{(0)} \equiv a_{2} \bar{y} e^{i\left(m \phi-\omega_{2}^{(0)} t\right)} .
$$

These are modes in which the fluid velocity is constant on torus cross-sections and entirely radial or vertical in the case of $W_{1}^{(0)}$ or $W_{2}^{(0)}$, respectively. These modes correspond to radial and vertical epicyclic oscillations of a test particle in a circular orbit. The correspondence between the fluid modes and test particle epicyclic oscillations can be confirmed by looking at the case of a general potential. Substituting equation (35) into the Papaloizou-Pringle equation (32), we find that they are still solutions of the more general problem. The corresponding eigenfrequencies in the corotating frame $\left(\omega-m \Omega_{0}\right)$ are indeed the radial and vertical epicyclic frequencies $\omega_{r}$ and $\omega_{z}$ in the case of $W_{r}$ and $W_{z}$, respectively.

\subsection{First Order Perturbation Theory}

We now expand the general eigenvalue problem for thick tori in a power series in $\beta$ :

$$
\begin{gathered}
W=W^{(0)}+\beta W^{(1)}+\beta^{2} W^{(2)}+\ldots \\
\bar{\omega}=\bar{\omega}^{(0)}+\beta \bar{\omega}^{(1)}+\beta^{2} \bar{\omega}^{(2)}+\ldots \\
f=f^{(0)}+\beta f^{(1)}+\beta^{2} f^{(2)}+\ldots \\
\bar{\Omega}=1+\beta \bar{\Omega}^{(1)}+\beta^{2} \bar{\Omega}^{(2)}+\ldots \\
\hat{L}=\hat{L}^{(0)}+\beta \hat{L}^{(1)}+\beta^{2} \hat{L}^{(2)}+\ldots
\end{gathered}
$$

Then if we expand the Papaloizou-Pringle equation (26) to first order in $\beta$, we obtain

$$
\hat{L}^{(1)} W^{(0)}+\hat{L}^{(0)} W^{(1)}=-2 n \bar{\sigma}_{0}^{2} W^{(1)}-4 n \bar{\sigma}_{0}\left(\bar{\omega}^{(1)}-m \bar{\Omega}^{(1)}\right) W^{(0)} .
$$

We now expand $W^{(1)}$ in our orthonormal basis of zeroth order eigenfunctions,

$$
W^{(1)}=\sum_{j} b_{j} W_{j}^{(0)}
$$


and take the inner product of equation (41) with a particular eigenfunction $W_{J}^{(0)}$. If $J$ labels the particular mode of interest, then this gives us an equation for the first order correction to the eigenfrequency:

$$
\bar{\omega}^{(1)}=-\frac{1}{4 n \bar{\sigma}_{0}}\left[<W^{(0)}\left|\hat{L}^{(1)}\right| W^{(0)}>-4 n \bar{\sigma}_{0} m<W^{(0)} \mid \bar{\Omega}^{(1)} W^{(0)}>\right] .
$$

If $J$ labels a different mode from the one of interest, then we get an equation for the complex coefficients in the first order eigenfunction:

$$
b_{J}=\frac{1}{2 n\left(\bar{\sigma}_{0 J}^{2}-\bar{\sigma}_{0}^{2}\right)}\left[<W_{J}^{(0)}\left|\hat{L}^{(1)}\right| W^{(0)}>-4 n \bar{\sigma}_{0} m<W_{J}^{(0)} \mid \bar{\Omega}^{(1)} W^{(0)}>\right] .
$$

Now, for a constant specific angular momentum torus,

$$
\bar{\Omega}=\frac{\ell_{0}}{r^{2} \Omega_{0}}=\frac{r_{0}^{2}}{r^{2}}=(1+\beta \bar{x})^{-2} .
$$

Hence, we find $\bar{\Omega}^{(1)}=-2 \bar{x}$. The first order correction to the equipotential function is given by

$$
f^{(1)}=-\frac{\bar{x}^{3}}{3} \overline{\mathcal{U}}_{r r r}-\bar{x} \bar{y}^{2} \overline{\mathcal{U}}_{r z z}
$$

where

$$
\overline{\mathcal{U}}_{r r r} \equiv \frac{r_{0}}{\Omega_{0}^{2}}\left(\frac{\partial^{3} \mathcal{U}}{\partial r^{3}}\right)_{0} \text { and } \overline{\mathcal{U}}_{r z z} \equiv \frac{r_{0}}{\Omega_{0}^{2}}\left(\frac{\partial^{3} \mathcal{U}}{\partial r \partial z^{2}}\right)_{0}
$$

Finally, the first order correction to $\hat{L}$ is given by

$$
\hat{L}^{(1)}=f^{(1)} \frac{\partial^{2}}{\partial \bar{x}^{2}}+\left(f^{(0)}+n \frac{\partial f^{(1)}}{\partial \bar{x}}\right) \frac{\partial}{\partial \bar{x}}+f^{(1)} \frac{\partial^{2}}{\partial \bar{y}^{2}}+n \frac{\partial f^{(1)}}{\partial \bar{y}} \frac{\partial}{\partial \bar{y}} .
$$

Hence for the radial epicyclic mode, $W_{1}^{(0)}=a_{1} \bar{x}$, we have

$$
\hat{L}^{(1)} \mid W_{1}^{(0)}>=a_{1}\left[1-\left(\bar{\omega}_{r}^{2}+n \overline{\mathcal{U}}_{r r r}\right) \bar{x}^{2}-\left(\bar{\omega}_{z}^{2}+n \overline{\mathcal{U}}_{r z z}\right) \bar{y}^{2}\right]
$$

and

$$
\bar{\Omega}^{(1)} \mid W_{1}^{(0)}>=-2 a_{1} \bar{x}^{2} .
$$

On the other hand, for the vertical epicyclic mode, $W_{2}^{(0)}=a_{2} \bar{y}$,

$$
\hat{L}^{(1)} \mid W_{2}^{(0)}>=-2 n a_{2} \overline{\mathcal{U}}_{r z z} \bar{x} \bar{y}
$$

and

$$
\bar{\Omega}^{(1)} \mid W_{2}^{(0)}>=-2 a_{2} \bar{x} \bar{y} .
$$


In both cases, we immediately see from equation (43) that the first order correction to the frequency vanishes.

For the radial epicyclic mode, equations (44), (49) and (50) give three nonzero coefficients for the first order corrections to the eigenfunction, corresponding to the $J=0,4$, and 5 eigenmodes of Table 1. Adding these together, the resulting radial epicyclic eigenfunction $W_{1}$ turns out to be given by

$$
\begin{aligned}
\frac{W_{1}}{a_{1}}= & \bar{x}+\frac{\beta}{2 \bar{\omega}_{r}^{4}\left[(2 n+3) \bar{\omega}_{z}^{2}-(n+1) \bar{\omega}_{r}^{2}\right]}\left\{\left(2 \bar{\omega}_{z}^{2}-\bar{\omega}_{r}^{2}\right)\left(\overline{\mathcal{U}}_{r r r}-\bar{\omega}_{r}^{2} \mp 8 m \bar{\omega}_{r}\right)+\bar{\omega}_{r}^{2} \overline{\mathcal{U}}_{r z z}\right. \\
& +\left[\left(2 \bar{\omega}_{z}^{2}-\bar{\omega}_{r}^{2}\right)\left(-\bar{\omega}_{r}^{2} \pm 8 m n \bar{\omega}_{r}-n \overline{\mathcal{U}}_{r r r}\right) \pm 8 m \bar{\omega}_{r} \bar{\omega}_{z}^{2}-\bar{\omega}_{z}^{2} \overline{\mathcal{U}}_{r r r}+\bar{\omega}_{r}^{2} \overline{\mathcal{U}}_{r z z}\right] \bar{\omega}_{r}^{2} \bar{x}^{2} \\
& \left.+\left[\bar{\omega}_{z}^{2} \overline{\mathcal{U}}_{r r r}-\bar{\omega}_{r}^{2} \bar{\omega}_{z}^{2} \mp 8 m \bar{\omega}_{r} \bar{\omega}_{z}^{2}-(n+1) \bar{\omega}_{r}^{2} \overline{\mathcal{U}}_{r z z}\right] \bar{\omega}_{r}^{2} \bar{y}^{2}\right\}+\mathcal{O}\left(\beta^{2}\right),
\end{aligned}
$$

where $a_{1}$ is the normalization constant from Table 1 .

The vertical epicyclic mode is much simpler. Equations (444), (51) and (52) give only

one nonzero coefficient, corresponding to the $J=3$ eigenmode in Table 1 . The resulting eigenfunction is then given by

$$
\frac{W_{2}}{a_{2}}=\bar{y}+\frac{\beta}{\bar{\omega}_{r}^{2}}\left( \pm 4 m \bar{\omega}_{z}-\overline{\mathcal{U}}_{r z z}\right) \bar{x} \bar{y}+\mathcal{O}\left(\beta^{2}\right)
$$

\subsection{Second Order Perturbation Theory}

Because the first order frequency corrections vanish, we have to go to second order to determine the finite pressure corrections to the epicyclic mode frequencies.

The second order expansion terms are $\bar{\Omega}^{(2)}=3 \bar{x}^{2}$,

$$
f^{(2)}=-\frac{1}{12}\left(\bar{x}^{4} \overline{\mathcal{U}}_{r r r r}+6 \bar{x}^{2} \bar{y}^{2} \overline{\mathcal{U}}_{r r z z}+\bar{y}^{4} \overline{\mathcal{U}}_{z z z z}\right)
$$

and

$$
\hat{L}^{(2)}=f^{(2)} \frac{\partial^{2}}{\partial \bar{x}^{2}}+\left(f^{(1)}-\bar{x} f^{(0)}+n \frac{\partial f^{(2)}}{\partial \bar{x}}\right) \frac{\partial}{\partial \bar{x}}+f^{(2)} \frac{\partial^{2}}{\partial \bar{y}^{2}}+n \frac{\partial f^{(2)}}{\partial \bar{y}} \frac{\partial}{\partial \bar{y}}-m^{2} f^{(0)} .
$$

Here

$$
\overline{\mathcal{U}}_{r r r r} \equiv \frac{r_{0}^{2}}{\Omega_{0}^{2}}\left(\frac{\partial^{4} \mathcal{U}}{\partial r^{4}}\right)_{0}, \quad \overline{\mathcal{U}}_{r r z z} \equiv \frac{r_{0}^{2}}{\Omega_{0}^{2}}\left(\frac{\partial^{4} \mathcal{U}}{\partial r^{2} \partial z^{2}}\right)_{0}, \quad \text { and } \quad \overline{\mathcal{U}}_{z z z z} \equiv \frac{r_{0}^{2}}{\Omega_{0}^{2}}\left(\frac{\partial^{4} \mathcal{U}}{\partial z^{4}}\right)_{0}
$$

The second-order terms in the Papaloizou-Pringle equation (26) give

$$
\hat{L}^{(2)} W^{(0)}+\hat{L}^{(1)} W^{(1)}+\hat{L}^{(0)} W^{(2)}=-2 n \bar{\sigma}_{0}^{2} W^{(2)}-4 n \bar{\sigma}_{0}\left(\bar{\omega}^{(1)}-m \bar{\Omega}^{(1)}\right) W^{(1)}
$$




$$
-4 n \bar{\sigma}_{0}\left(\bar{\omega}^{(2)}-m \bar{\Omega}^{(2)}\right) W^{(0)}-2 n\left(\bar{\omega}^{(1)}-m \bar{\Omega}^{(1)}\right)^{2} W^{(0)}
$$

Once again, we expand the second order eigenfunction in terms of the zeroth order eigenfunctions,

$$
W^{(2)}=\sum_{j} c_{j} W_{j}^{(0)} .
$$

Then on taking the inner product of equation (58) with the zeroth order eigenfunction of the mode of interest, we find that the second order correction to the frequency is (for the case when the first order correction $\bar{\omega}^{(1)}$ vanishes)

$$
\begin{aligned}
\bar{\omega}^{(2)}= & -\frac{1}{4 n \bar{\sigma}_{0}}\left\{<W^{(0)}\left|\left[\hat{L}^{(2)}-4 n \bar{\sigma}_{0} m \bar{\Omega}^{(2)}+2 n m^{2}\left(\bar{\Omega}^{(1)}\right)^{2}\right]\right| W^{(0)}>\right. \\
& \left.+\sum_{j} b_{j}<W^{(0)}\left|\left[\hat{L}^{(1)}-4 n m \bar{\sigma}_{0} \bar{\Omega}^{(1)}\right]\right| W_{j}^{(0)}>\right\} .
\end{aligned}
$$

For the radial epicyclic mode, we find

$$
\begin{aligned}
\bar{\omega}_{1}= & \pm \bar{\omega}_{r}+m \mp \frac{\beta^{2}}{8(n+2) \bar{\omega}_{r}^{5} \bar{\omega}_{z}^{2}}\left\{-\bar{\omega}_{r}^{2} \bar{\omega}_{z}^{2} \overline{\mathcal{U}}_{r r r r}-\bar{\omega}_{r}^{4} \overline{\mathcal{U}}_{r r z z}-2 m^{2} \bar{\omega}_{r}^{4} \bar{\omega}_{z}^{2}+\frac{1}{\left[(2 n+3) \bar{\omega}_{z}^{2}-(n+1) \bar{\omega}_{r}^{2}\right]} \times\right. \\
\times & \left\{2 \bar{\omega}_{r}^{4} \bar{\omega}_{z}^{2}\left[(n+2) \bar{\omega}_{r}^{2}-(2 n+5) \bar{\omega}_{z}^{2}\right]+2 \bar{\omega}_{r}^{2} \bar{\omega}_{z}^{2} \overline{\mathcal{U}}_{r r r}\left[(n-1) \bar{\omega}_{r}^{2}-(2 n-1) \bar{\omega}_{z}^{2}\right]+4 \bar{\omega}_{r}^{4} \bar{\omega}_{z}^{2} \overline{\mathcal{U}}_{r z z}\right. \\
& +\bar{\omega}_{z}^{2} \overline{\mathcal{U}}_{r r r}^{2}\left[(5+6 n) \bar{\omega}_{z}^{2}-(3 n+1) \bar{\omega}_{r}^{2}\right]+\bar{\omega}_{r}^{2} \overline{\mathcal{U}}_{r r r} \overline{\mathcal{U}}_{r z z}\left[(2 n-1) \bar{\omega}_{z}^{2}-(n+1) \bar{\omega}_{r}^{2}\right]+2 \bar{\omega}_{r}^{4}(n+1) \overline{\mathcal{U}}_{r z z}^{2} \\
& \pm 4 m \bar{\omega}_{r}^{3} \bar{\omega}_{z}^{2}\left[(7 n+15) \bar{\omega}_{r}^{2}-(14 n+37) \bar{\omega}_{z}^{2}\right] \pm 4 m \bar{\omega}_{r} \bar{\omega}_{z}^{2} \overline{\mathcal{U}}_{r r r}\left[(7 n-1) \bar{\omega}_{r}^{2}-(14 n+5) \bar{\omega}_{z}^{2}\right] \\
& \left.\left. \pm 4 m \bar{\omega}_{r}^{3} \overline{\mathcal{U}}_{r z z}\left[(n+1) \bar{\omega}_{r}^{2}-(2 n-5) \bar{\omega}_{z}^{2}\right]+8 m^{2} \bar{\omega}_{r}^{2} \bar{\omega}_{z}^{2}\left[(9-7 n) \bar{\omega}_{r}^{2}+(14 n-11) \bar{\omega}_{z}^{2}\right]\right\}\right\} \\
& +\mathcal{O}\left(\beta^{3}\right) .
\end{aligned}
$$

The frequency of the vertical epicyclic mode is

$$
\begin{aligned}
\bar{\omega}_{2}= & \pm \bar{\omega}_{z}+m \mp \frac{\beta^{2}}{8 \bar{\omega}_{r}^{4} \bar{\omega}_{z}^{3}(n+2)}\left\{-\bar{\omega}_{r}^{2} \bar{\omega}_{z}^{2} \overline{\mathcal{U}}_{r r z z}-\bar{\omega}_{r}^{4} \overline{\mathcal{U}}_{z z z z}-2 \bar{\omega}_{r}^{2} \bar{\omega}_{z}^{2} \overline{\mathcal{U}}_{r z z}\right. \\
& +\overline{\mathcal{U}}_{r z z}\left[\bar{\omega}_{z}^{2} \overline{\mathcal{U}}_{r r r}+\left(2 \bar{\omega}_{z}^{2}+3 \bar{\omega}_{r}^{2}\right) \overline{\mathcal{U}}_{r z z}\right] \mp 4 m \bar{\omega}_{z}\left[\bar{\omega}_{r}^{2} \bar{\omega}_{z}^{2}+\bar{\omega}_{z}^{2} \overline{\mathcal{U}}_{r r r}+\left(4 \bar{\omega}_{z}^{2}+3 \bar{\omega}_{r}^{2}\right) \overline{\mathcal{U}}_{r z z}\right] \\
& \left.+2 m^{2} \bar{\omega}_{z}^{2}\left(16 \bar{\omega}_{z}^{2}+4 \bar{\omega}_{r}^{2}-\bar{\omega}_{r}^{4}\right)\right\}+\mathcal{O}\left(\beta^{3}\right) .
\end{aligned}
$$

\subsection{Spherically Symmetric Point Mass Potential}

In this case we have

$$
\bar{\omega}_{r}^{2}=\bar{\omega}_{z}^{2}=1, \quad \overline{\mathcal{U}}_{r r r}=-6, \quad \overline{\mathcal{U}}_{r z z}=-3,
$$




$$
\overline{\mathcal{U}}_{r r r r}=36, \quad \overline{\mathcal{U}}_{r r z z}=12, \quad \text { and } \quad \overline{\mathcal{U}}_{z z z z}=-9 .
$$

For the radial epicyclic mode,

$$
\bar{\omega}_{1}= \pm 1+m \mp \frac{\beta^{2}}{4(n+2)^{2}}\left(53 n+6 \pm 76 n m \mp 8 m+27 n m^{2}-10 m^{2}\right)+\mathcal{O}\left(\beta^{3}\right)
$$

and

$$
\begin{aligned}
W_{1}=\left[\frac{2 n(n+1)}{\pi}\right]^{1 / 2}\{\bar{x}+ & \frac{\beta}{n+2}\left[-5 \mp 4 m+(3 n+1 \pm 4 m \pm 4 n m) \bar{x}^{2}\right. \\
& \left.\left.+\left(\frac{3}{2} n-2 \mp 4 m\right) \bar{y}^{2}\right]\right\}+\mathcal{O}\left(\beta^{2}\right) .
\end{aligned}
$$

For the vertical epicyclic mode,

$$
\bar{\omega}_{2}= \pm 1+m \mp \frac{\beta^{2}}{4(n+2)}\left(33 \pm 52 m+19 m^{2}\right)+\mathcal{O}\left(\beta^{3}\right)
$$

and

$$
W_{2}=\left[\frac{2 n(n+1)}{\pi}\right]^{1 / 2}[\bar{y}+\beta(3 \pm 4 m) \bar{x} \bar{y}]+\mathcal{O}\left(\beta^{2}\right) .
$$

It is perhaps interesting to note that for $n=3$ (i.e. radiation pressure dominated) tori, the axisymmetric $(m=0)$ radial and vertical epicyclic modes frequencies continue to be degenerate to the $\beta^{2}$ order of accuracy.

\subsection{Pseudo-Newtonian Potential}

In the case of the Paczyński \& Wiita (1980) pseudo-Newtonian potential, in units where $c=G=M=1$,

$$
\Phi=-\frac{1}{\left(r^{2}+z^{2}\right)^{1 / 2}-2}
$$

we have

$$
\begin{aligned}
\bar{\omega}_{r}^{2} & =\frac{r_{0}-6}{r_{0}-2}, \quad \bar{\omega}_{z}^{2}=1, \quad \overline{\mathcal{U}}_{r r r}=-\frac{6\left(r_{0}^{2}-8 r_{0}+8\right)}{\left(r_{0}-2\right)^{2}}, \\
\overline{\mathcal{U}}_{r z z} & =-\frac{3 r_{0}-2}{r_{0}-2}, \quad \overline{\mathcal{U}}_{r r r r}=\frac{12\left(3 r_{0}^{3}-30 r_{0}^{2}+60 r_{0}-40\right)}{\left(r_{0}-2\right)^{3}} \\
\overline{\mathcal{U}}_{r r z z} & =\frac{4\left(3 r_{0}^{2}-4 r_{0}+2\right)}{\left(r_{0}-2\right)^{2}}, \quad \text { and } \quad \overline{\mathcal{U}}_{z z z z}=-\frac{3\left(3 r_{0}-2\right)}{r_{0}-2}
\end{aligned}
$$

These expressions may be substituted into equations (53), (54), (61) and (62) to obtain rather complicated, explicit expressions for the epicyclic mode eigenfunctions and eigenfrequencies as a function of location of the torus pressure maximum and $\beta$. Rather than present those expressions here, we use them to numerically evaluate the mode frequencies. 


\subsubsection{Axisymmetric Modes}

Figure 2 shows the behavior of the axisymmetric $(m=0)$ radial and vertical epicyclic mode frequencies. The $\beta=0$ curves in these Figures correspond to the test particle frequencies. For a given pressure maximum radius $r_{0}$, there is a maximum value of $\beta$ beyond which equilibrium tori cannot exist. This limit is indicated by the dashed curves in the Figures.

Note from Figure 2 that the axisymmetric vertical epicyclic mode frequency for nonslender tori exhibits a maximum value as a function of $r_{0}$, in contrast to the behavior of the test particle frequency in a pseudo-Newtonian potential. This gives rise to interesting behavior of the ratio of the vertical to radial mode frequencies, as shown in Figure 3, Once again, the dashed line indicates the limit beyond which equilibrium tori can exist. For small values of $\beta$ the ratio of test particle frequencies rises monotonically toward smaller radii, and there is therefore a unique radius at which the frequency ratio can take on any specific value. The dotted line shows the 3:2 frequency ratio that is found in the high frequency QPOs. As the torus thickens, the radius at which the 3:2 commensurability occurs moves inward, and as shown in Figure 4 (the left part), each of the mode frequencies increases. This continues until $\beta=0.134589$, when suddenly tori at two different radii display a 3:2 commensurability between the axisymmetric epicyclic modes. The inner torus produces higher frequencies than the outer torus. As $\beta$ increases further, these two tori move toward each other, converging at $\beta=0.138079$. For still thicker tori, there is no radius at which the axisymmetric epicyclic modes are in a 3:2 ratio. Hence we conclude that the axisymmetric epicyclic modes can represent both observed high frequency QPOs only if the torus is not too thick. The right part of Figure 4 shows the equipotential surfaces of the torus that exhibits the highest such mode frequencies while still retaining the 3:2 commensurability.

The analytic, poloidal velocity fields of the axisymmetric radial and vertical epicyclic modes of this nonslender torus are shown in Figure 5. The radial mode involves some vertical expansion on outward displacements (and corresponding vertical compression on inward displacements). The reason for this is that the vertical tidal gravity is less at larger radii, and so the torus expands under vertical pressure gradients. For the vertical epicyclic mode, the motions of at least the outer parts of the torus are also easy to understand. The upper half of the torus moves radially outward (and the lower half moves radially inward) on upward vertical displacements. This is because the radial component of the gravitational field decreases as one moves off the midplane, and so centrifugal and pressure gradient accelerations drive outward radial displacements in the upper half of the torus. Similarly the increasing gravity as the lower half moves toward the midplane causes inward

radial displacements. The pressure forces exerted by these radial motions act to oppose the vertical motions of the inner parts of the torus. This effect is so strong that the inner parts of 
the torus shown in the Figure are actually oscillating in the opposite direction to the zeroth order vertical epicyclic mode. Our perturbation expansion may therefore not be accurate for this thick a torus. It nevertheless makes sense that the vertical displacements in the vertical epicyclic mode of a radially extended torus cannot in general all be in the same direction simultaneously, as there are no forces that would maintain such radial coherence.

Increasing the thickness of the torus (increasing $\beta$ ) always decreases the axisymmetric mode frequencies so that they are less than the test particle frequencies. Our complicated analytic formulas for the mode frequencies suggest that the physical reasons for the details of this decrease must itself be complicated. However, it is not hard to guess physically why the mode frequencies should decrease in general. First, unless the radius of the pressure maximum is very close to the innermost stable circular orbit, the center of mass of a nonslender torus always lies outside the radius of the pressure maximum. At large radii both of the test particle frequencies always decrease with radius. Hence the outward shift of the center of mass of the torus as it thickens should alone decrease the frequency of the epicyclic modes. At small radii, the test particle radial epicyclic frequency increases with radius, and so one might expect that the radial epicyclic mode frequency would increase with torus thickness (except perhaps when the torus is very close to the innermost stable circular orbit and the center of mass then lies inside the pressure maximum). However, in this regime the mode frequency appears to depend primarily on the nearby presence of the cusp in the equipotential surfaces. There is no radial restoring force at the cusp, and as a result the radial epicyclic mode frequency of a nonslender torus near the cusp drops dramatically, as shown in Figure 2. Finally, even when the pressure maximum of the torus is close to the innermost stable circular orbit $r \lesssim 6.5 \mathrm{M}$ so that the center of mass of the torus moves inward on thickening, the vertical epiyclic mode is still biased toward the outer parts of the torus because pressure forces reduce the vertical oscillations in the inner parts. As a result, the frequency of the vertical epicyclic mode still decreases with torus thickness even in this regime.

\subsubsection{Nonaxisymmetric Modes with $m= \pm 1$}

Recently, Bursa (2006) suggested a new pair of modes having the 3:2 commensurability, that would better satisfy the observational constraints on black hole spins and masses, in particular the most recent spin estimates from spectral fitting to the X-ray spectrum of GRO J1655-40 (Shafee et al. 2006; Davis, Done, \& Blaes 2006). These modes are the axisymmetric vertical and the nonaxisymmetric $m=-1$ radial mode, whose test particle frequencies occur in a 3:2 ratio close to the marginally stable orbit.

The nonaxisymmetric $(m= \pm 1)$ radial mode frequencies are shown in Figures 6 and 7 . 
Again, the curves with $\beta=0$ correspond to the frequency of a test particle, and the dashed line denotes the limit for tori in equilibrium. Figure 6 shows the sum of the axisymmetric radial frequency and $\Omega_{0}$ (i.e., the $m=1$ mode) for different torus thicknesses. With increasing values of $\beta$, the sum of the frequencies slightly decreases. The situation becomes more complicated for the $m=-1$ mode. The frequency difference between $\Omega_{0}$ and the axisymmetric radial epicyclic frequency first increases with growing $\beta$, but then it decreases with increasing $\beta$ in the interval $10.895 M<r<15.573 M$ (Figure 7). Finally, for radii greater than $15.573 M$, the frequency difference increases with $\beta$ again.

The ratio of the axisymmetric vertical frequency to the $m=-1$ radial mode frequency for different torus thicknesses can be seen in the left panel of Figure 8 . Unlike the resonance for the axisymmetric epicyclic modes, the radius at which these two modes are in a $3: 2$ ratio moves outward with increasing $\beta$. This corresponds to a decrease of the individual frequencies, as can be seen in the right part of the Figure, and the frequencies eventually become zero for $\beta=\beta_{\max }=0.725108$. For tori with $\beta<\beta_{\max }$, there exists exactly one torus pressure maximum location that generates a 3:2 frequency ratio, and no such location exists for still thicker tori corresponding to $\beta \geq \beta_{\max }$. This would suggest that even fairly large tori can exhibit a 3:2 resonance, but the perturbative method is valid only for small $\beta$, and any firm results on very extended tori must be obtained by other methods.

\subsection{Consequences for Black Hole Spin Estimates}

The first black hole spin estimates from fitting the axisymmetric epicyclic mode frequencies to the observed QPOs from GRO J1655-40 were reported by Abramowicz \& Kluźniak (2001). Recently Török et al. (2005) have used the orbital resonance model to estimate the spins of all three microquasars with known masses. The most tightly constrained spin was in GRO J1655-40, where $a / M$ was found to lie between 0.93 and 0.99 . This spin value that was derived from a resonance between the axisymmetric epicyclic modes is not in accord with the independent spin measurements, determined by fitting the X-ray spectral continua: $a / M \sim 0.65-0.75$ (Shafee et al. 2006), $a / M \sim 0.62$ or less if the disk inclination is free (Davis, Done, \& Blaes 2006) for GRO J1655-40.

However, the resonance model estimates were based on the epicyclic frequencies for free test particles. Figure 4 shows that the axisymmetric epicyclic frequencies at the resonant radius will be higher for a nonslender torus than for a test particle. The maximal frequency shift due to pressure effects is around 15 percent of the test particle frequency. The previous studies have therefore most probably overestimated the actual value of the spin of the black hole, which explains some of the discrepancies with other methods. 
On the other hand a resonance between the axisymmetric vertical epicyclic mode and the nonaxisymmetric $m=-1$ radial mode yields a black hole spin for GRO J1655-40 (Bursa 2006) compatible with other recent spin estimates although Bursa used the frequencies for free particles. As seen from Figure 8, the frequencies of these two modes at the resonant radius decrease with growing torus thickness, contrary to the axisymmetric modes. Therefore in this case the black hole spins should be higher than previously estimated. The maximal shift of the frequencies (and consequently the black hole spin) can be very large, but our perturbative method is valid for small $\beta$, and is not reliable for $\beta \approx 0.7$.

The above modifications of the spin estimates are based on purely Newtonian calculations using the Paczyński-Wiita gravitational potential. In order to explore the real behavior of thick tori orbiting around rotating black holes, one needs to solve the hydrodynamical equations in the Kerr metric.

\subsection{Comparison with Numerical Simulation}

We compare our analytically calculated frequencies for the axisymmetric epicyclic modes with those from hydrodynamic numerical simulations. The numerical simulations were

performed using the ZEUS-2D code by Stone \& Norman (1992). The radial, and vertical epicyclic oscillations, respectively, were excited by applying a purely radial, or vertical constant velocity field perturbation, respectively, to the torus at $\mathrm{t}=0$. Then we Fourier analyzed the radial and vertical positions of the center of mass in order to find the oscillatory frequencies.

Figure 9 compares the results of our analytic expressions (64) and (66) with $m=0$ to the epicyclic mode frequencies measured from the simulations as a function of torus thickness. The agreement is excellent for $\beta \lesssim 0.2$. Figure 10 similarly compares the analytic $m=0$ frequencies from our perturbation theory to the simulation results for tori in pseudoNewtonian potentials. Once again, there is good agreement for $\beta \lesssim 0.2$. The general trend that the frequencies decrease with increasing $\beta$ was also observed by Rubio-Herrera \& Lee (2005) in their simulations.

\section{Discussion and Conclusions}

In this paper we have assumed that (possibly nonaxisymmetric) vertical and radial epicyclic modes of fluid tori are responsible for the commensurate pairs of high frequency QPOs in black hole X-ray binaries. We have derived exact analytic formulae for the mode 
eigenfunctions and frequencies. We find that the frequencies are always below those corresponding to free test particles, an effect that must be taken into account before identifying such modes with observed QPOs.

While our analytic calculations are exact, they apply to very simplified and idealized configurations:

(1) Our calculations were restricted to Newtonian mechanics. This is a necessary first step to doing the fully general relativistic calculation in Kerr spacetime (Straub et al. 2007, in preparation). Moreover, Newtonian potentials that mock up various aspects of the physics of Schwarzschild spacetime are used by many numericists (e.g. Lee, Abramowicz \& Kluźniak 2004; Machida et al. 2006), and our results may be applied directly to their simulations.

(2) We considered isolated, non-accreting, non-magnetized, polytropic tori. The flow configuration of the accretion disks in X-ray binaries when they exhibit QPOs is still far from clear, but pressure-supported torus-like configurations remain an interesting possibility. Such configurations are seen as "inner tori" in global MRI simulations (De Villiers, Hawley \& Krolik 2003; Machida et al. 2006; Matsumoto \& Machida 2007). These inner tori have magnetic energy densities much less than the gas internal energy density, so magnetic fields are less important to the overall hydrostatic structure than gas pressure. (Strongly magnetized configurations may however exist in real systems, and our treatment in this paper would not be valid for them.) Among all the possible modes of an isolated torus, the epicyclic modes will be most robust to boundary conditions, as they correspond to physical displacements of the entire torus. We therefore believe that our calculations for the corrections to the mode frequencies will be reasonably robust to these boundary conditions. The entropy distribution of these tori is completely unknown, and existing simulations tell us little as they do not have consistent thermodynamics. Nevertheless, the luminous accretion flows exhibiting high frequency QPOs are likely to be radiation pressure supported, and a polytropic configuration with $n=3$ may therefore be fairly close to reality.

(3) For mathematical reasons, we also restricted ourselves to constant specific angular momentum tori. Such angular momentum distributions appear unlikely to exist in nature, as global simulations of disks with MRI turbulence produce inner tori with angular momentum distributions which are closer to the test particle ("Keplerian") limit. (Note, however, that these simulations currently lack optically thick radiative cooling which is likely to determine the overall pressure support of the flow, and therefore departures from Keplerian angular momentum.) The Papaloizou-Pringle equation with non-constant specific angular momentum is not a self-adjoint eigenvalue problem, and so we do not have a complete set of orthogonal basis eigenfunctions in order to expand the perturbed modes around the slender torus limit. The perturbation equations using the Lagrangian displacement vector as dependent 
variable can be used to generate a complete set of orthogonal eigenfunctions, at least within Newtonian mechanics (Schenk et al. 2005), and this may enable an analytic calculation of the corrections to the mode frequencies for non-constant specific angular momentum tori. We note that fully general relativistic simulations in Kerr spacetime of non-constant specific angular momentum oscillating tori exhibit axisymmetric radial and vertical epicyclic mode frequencies which are below the test particle frequencies (Fragile 2006, private communication), in agreement with the qualitative results we have found here.

We view the results of the calculations presented here as a necessary tool for identifying the modes in fully 3D, time dependent numerical simulations of magnetohydrodynamic tori. A preliminary example of what can be achieved is provided by Bursa (2006), who used our results to identify the $(m=1)$ radial and vertical epicyclic modes in the recent numerical simulations by Machida et al. (2006). Once identified, our analytic expressions for the mode eigenfunctions may also be useful in trying to explain why such modes are excited in simulations. They may also be useful in making predictions for the observed X-ray modulation. As explored numerically by Bursa et al. (2004) and Schnittman \& Rezzolla (2006), gravitational redshifts, Doppler shifts, and lensing of X-ray photons emitted by tori oscillating in both the vertical and radial epicyclic modes can produce detectable oscillations in the predicted X-ray fluxes measured by an observer.

We thank Mami Machida and Michal Bursa for preparing Figure 1 for us. We very much thank these colleagues and also Didier Barret, Axel Brandenburg, Chris Fragile, Jiří Horák, Vladimír Karas, Shoji Kato, Michiel van der Klis, Jean-Pierre Lasota, William Lee, Ryoji Matsumoto, Jeff McClintock, Mariano Mendéz, Paola Rebusco, Ron Remillard, Giora Shaviv, Zdeněk Stuchlík, Gabriel Török, Roberto Vio, Bob Wagoner and Piotr Życki for comments, criticism, and several helpful suggestions. Our work was supported in part by the following grants: NSF's PHY99-07949 and AST03-07657, Nordita's 2005 Nordic Project awarded to M.A.A., Czech MSM 4781305903 and LC06014, and Polish 1P03D 005 30. The authors are also grateful to the following institutions for hosting them while much of this work was carried out: the Kavli Institute for Theoretical Physics in Santa Barbara, the Institut d'Astrophysique de Paris, Nordita in Copenhagen, and the N. Copernicus Astronomical Centre in Warsaw.

\section{REFERENCES}

Abramowicz, M.A., 2005, AN, 326, 782 
Abramowicz, M.A., Blaes, O.M., Horák, J., Kluźniak, W. \& Rebusco, P., 2006, Classical and Quantum Gravity, 23, 1689

Abramowicz, M.A. \& Kluźniak, W., 2001, A\&A, 374, L19

Blaes O. M., 1985, MNRAS, 216, 553

Blaes, O.M., Arras, P. \& Fragile, P.C., 2006, MNRAS, 369, 1235

Bursa, M., 2006, Ph. D. thesis, Charles Univ., Prague

Bursa, M., Abramowicz, M.A., Karas, V. \& Kluźniak, W., 2004, ApJ, 617, L45

Davis, S. W., Done, C., \& Blaes, O. M. 2006, ApJ, 647, 525

De Villiers, J.-P., Hawley, J. F., \& Krolik, J. H., 2003, ApJ, 599, 1238

Hawley, J. F., \& Balbus, S. A. 2002, ApJ, 573, 738

Kluźniak, W. \& Abramowicz, M.A., 2001a, Phys. Rev. Lett., submitted astro-ph/0105057

Kluźniak, W., \& Abramowicz, M. A. 2001b, Acta Phys. Pol. B, B32, 3605

Kluźniak, W. \& Abramowicz, M.A., 2002, A\&A, submitted astro-ph/0203314

Lee, W.H., Abramowicz, M.A. \& Kluźniak, W., 2004, ApJ, 603, L93

Machida, M., Nakamura K.E. \& Matsumoto, R., 2006, PASJ, 58, 193

Matsumoto, R. \& Machida, M., 2007, in IAU Symp. 238, Black Holes from Stars to Galaxies, ed. V. Karas \& G. Mat (Cambridge: Cambridge Univ. Press), 37

Nayfeh, A.H. \& Mook, D.A., 1979, Nonlinear Oscillations (New York: Wiley)

Paczyński, B. \& Wiita, P.J., 1980, A\&A, 88, 23

Papaloizou, J.C.B. \& Pringle, J.E., 1984, MNRAS, 208, 721

Remillard, R. A., \& McClintock, J. E. 2006, ARA\&A, 44, 49

Rezzolla, L., Yoshida, S’i., Maccarone, T. J. \& Zanotti, O., 2003, MNRAS, 344, L37

Rubio-Herrera, E. \& Lee, W.H., 2005, MNRAS, 362, 789

Schenk, A.K., Arras, P., Flanagan, E.E., Teukolsky, S.A. \& Wasserman, I., 2001, Phys. Rev. $\mathrm{D}, 65,024001$ 
Schnittman, J. D., \& Rezzolla, L. 2006, ApJ, 637, L113

Shafee, R., McClintock, J. E., Narayan, R., Davis, S. W., Li, L., \& Remillard, R. A. 2006, ApJ, 636, L113

Stone, J.M. \& Norman, M. L., 1992, ApJS, 80, 791

Strohmayer, T. E., 2001, ApJ, 552, L49

Tassoul, J.-L., 1978, Theory of Rotating Stars, Princeton University Press, Princeton

Török, G., Abramowicz, M.A., Kluźniak, W. \& Stuchlík, Z., 2005, A\&A, 436, 1

Zanotti, O., Rezzolla, L. \& Font, J.A., 2003, MNRAS, 341, 832 


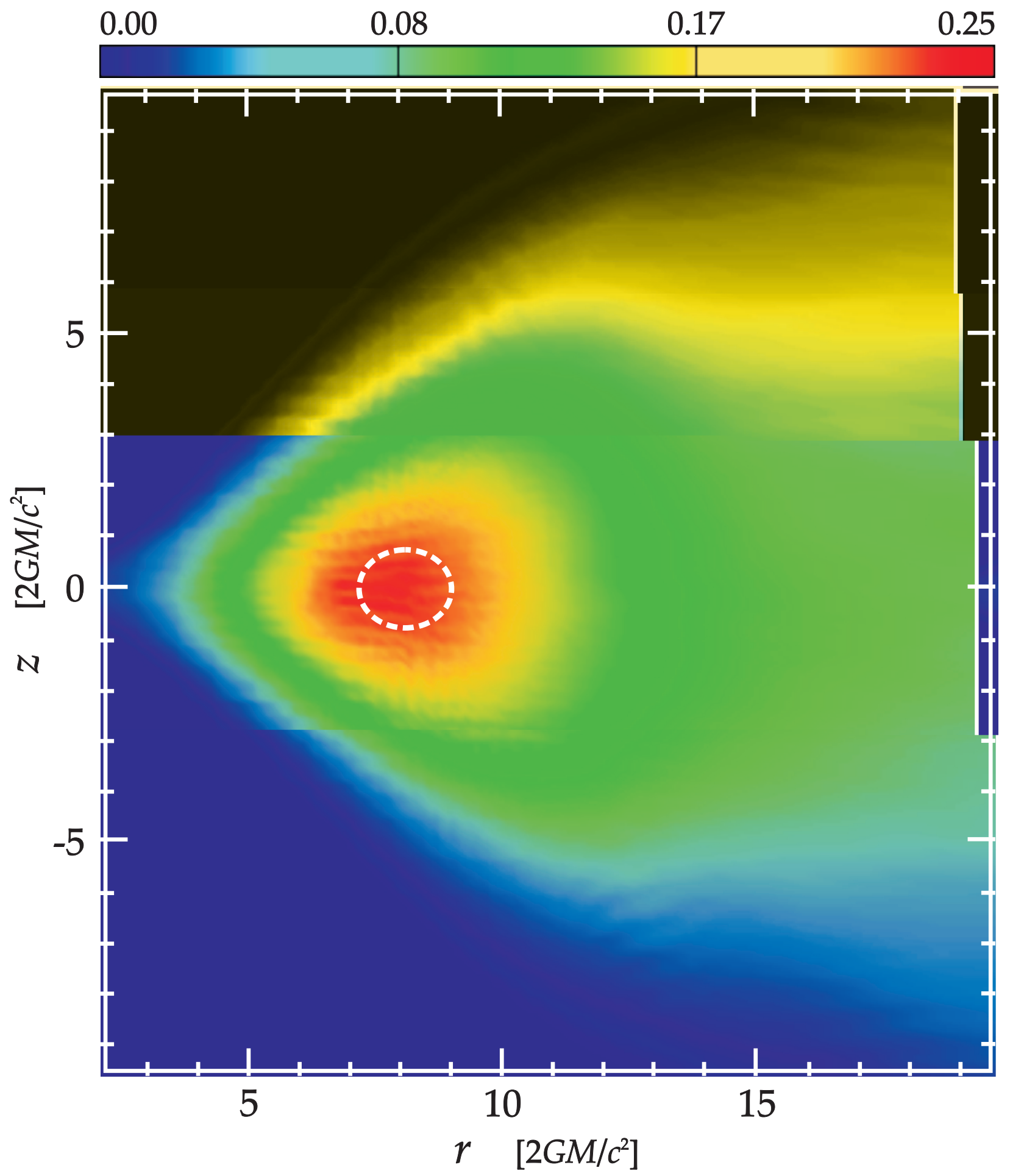

Fig. 1.- A pressure supported inner torus is apparent in the MHD simulations described by Machida et al. (2006) and Matsumoto \& Machida (2007). The relative density is color coded. The superimposed elliptical shape (a white broken line) corresponds to the analytic nonslender torus studied in this paper. 

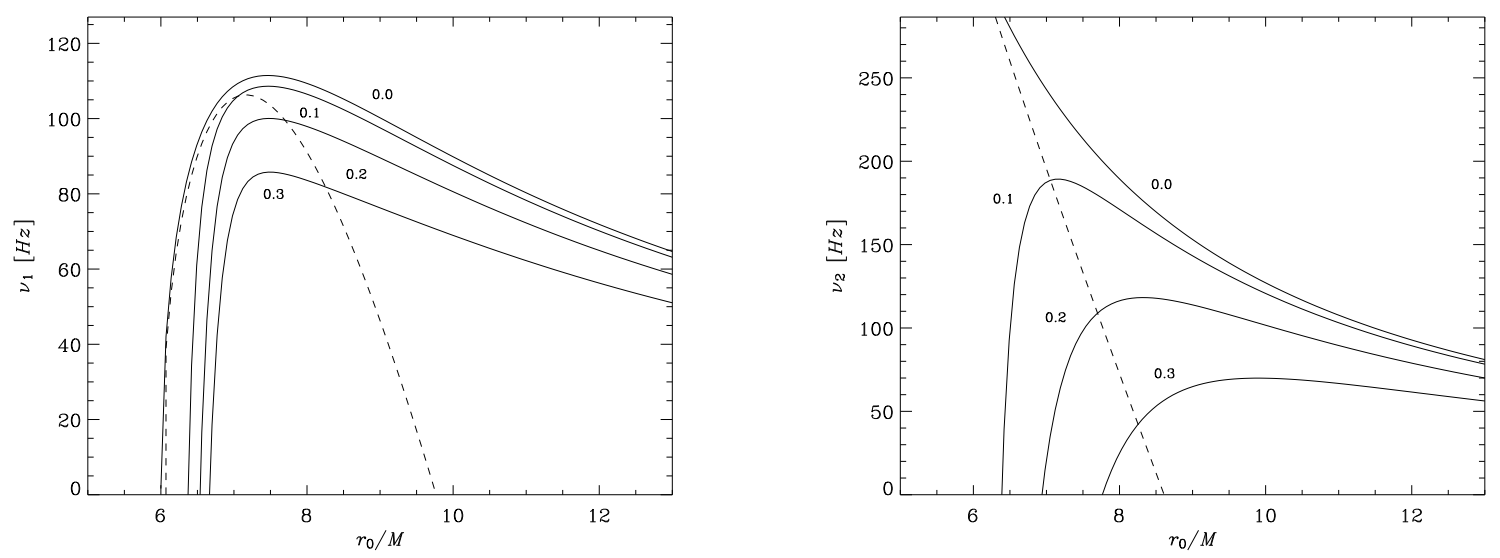

Fig. 2.- Left: Frequency of the axisymmetric $(m=0)$ radial epicyclic mode, $\nu_{1}=\omega_{1} /(2 \pi)$, for an $n=3$ torus in a pseudo-Newtonian potential with black hole mass $M=10 M_{\odot}$, as a function of radius of the torus pressure maximum in units of the gravitational radius. Different solid curves correspond to different values of the pressure parameter $\beta$, and are labeled as such. Models below the dashed curve are unphysical, as the corresponding tori extend outside the critical cusp equipotential. Right: Same as the left Figure, but for the axisymmetric vertical epicyclic mode. Once again, physical models lie above (to the right) of the dashed line. 


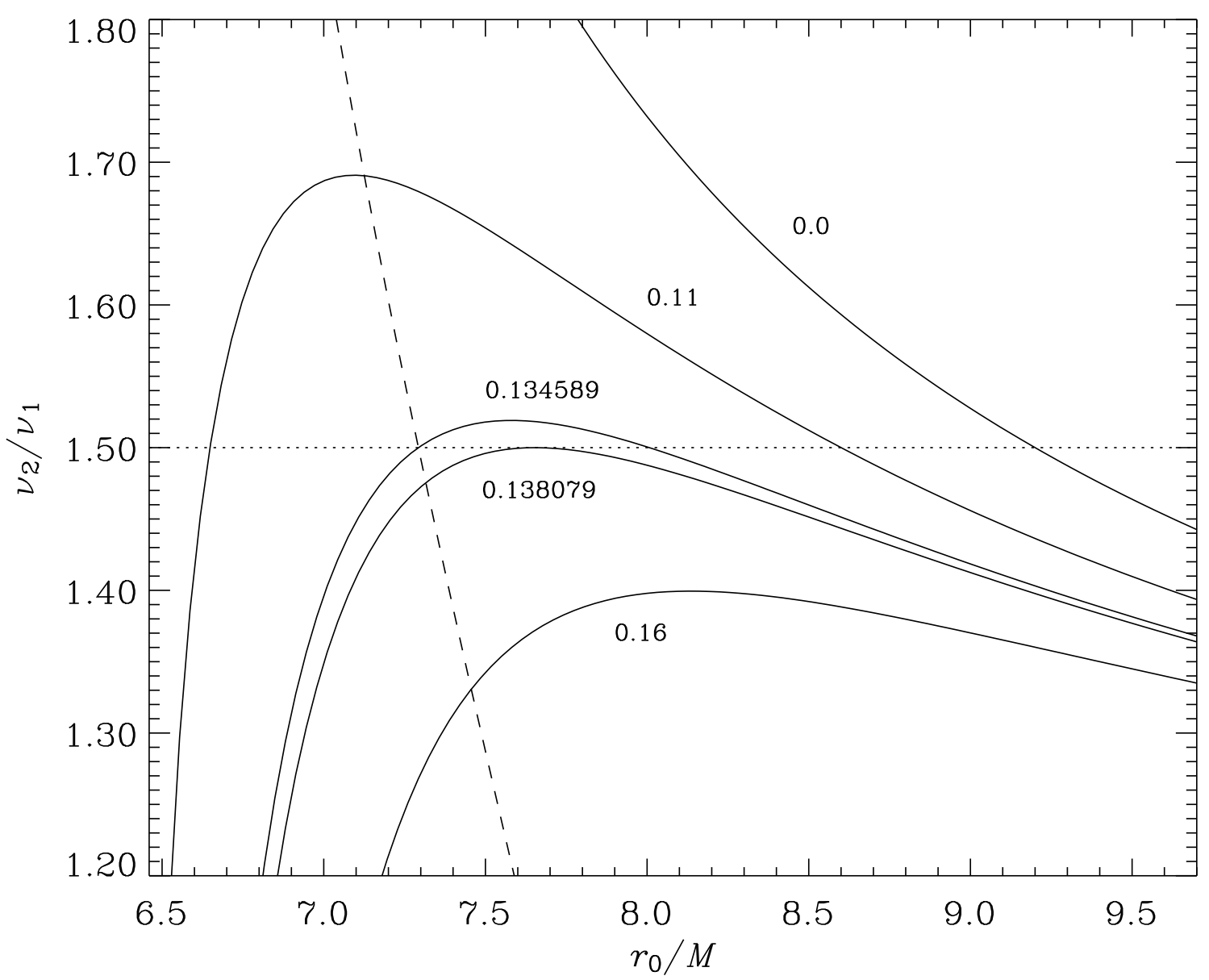

Fig. 3.- Ratio $\nu_{2} / \nu_{1}=\omega_{2} / \omega_{1}$ of the vertical to radial axisymmetric epicyclic mode frequencies for an $n=3$ torus in a pseudo-Newtonian potential, for different values of $\beta$ and as a function of radius of the torus pressure maximum. Again, physical models lie above and to the right of the dashed line. The dotted line indicates a 3:2 commensurability between the two modes. For a slender torus with a particular value of $\beta<0.134589$, there is one radius for which the axisymmetric mode frequency ratio will be 3:2. For somewhat thicker tori with values of $\beta$ satisfying $0.134589<\beta<0.138079 \equiv \beta_{\max }$, there are two locations of the torus pressure maximum where the mode frequencies are in a 3:2 ratio. No such radii exist for still thicker tori with $\beta>\beta_{\max }$. 

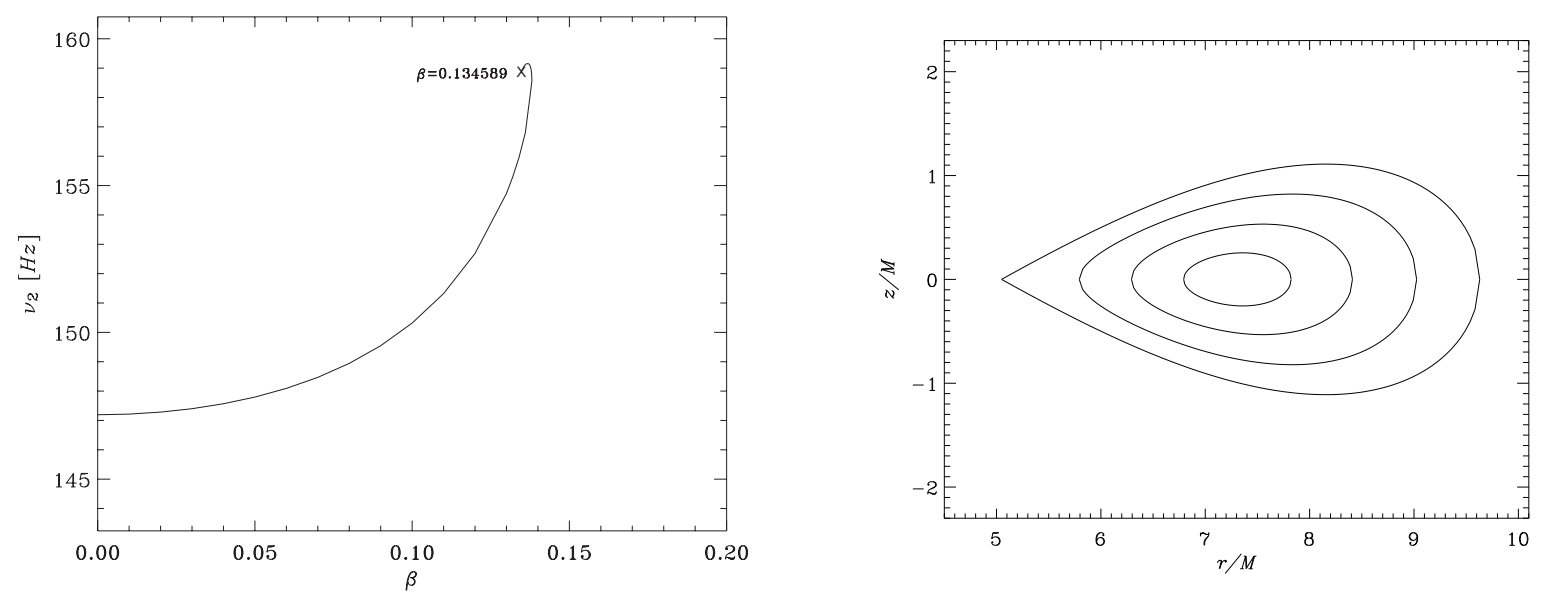

Fig. 4.- Left: Axisymmetric vertical epicyclic mode frequency $\nu_{2}=\omega_{2} /(2 \pi)$ for tori which have the axisymmetric vertical and radial modes in a 3:2 ratio, plotted as a function of $\beta$. Again, the tori are assumed to have $n=3$ and orbit in a pseudo-Newtonian potential with $M=10 M_{\odot}$. The $\beta=0.134589$ point in the Figure corresponds to the intersection of the dashed and dotted lines in Figure 3. Right: Meridional cross-section of a nonslender torus with $\beta=0.134589$ and a cusp at $r=5.0485 M$. The torus is centered at $r=7.293 M$ where the axisymmetric epicyclic mode frequencies $\omega_{1}$ and $\omega_{2}$ for this $\beta$ are in a $3: 2$ ratio. This case corresponds to the crossing point of the dashed and dotted lines in Figure 3 .
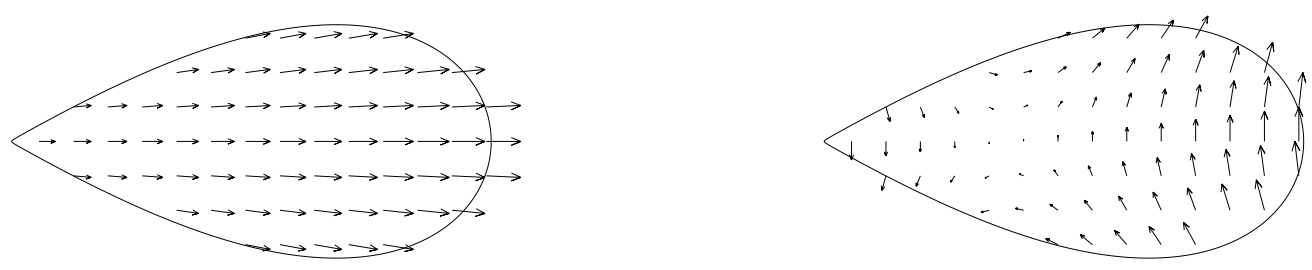

Fig. 5. - Poloidal velocity field for the radial epicyclic mode (left) and the vertical epicyclic mode (right) of a nonslender $n=3$ torus with $\beta=0.134589$ and pressure maximum at $r=7.293 M$ (the same torus illustrated in the right hand panel of Fig. (4). 


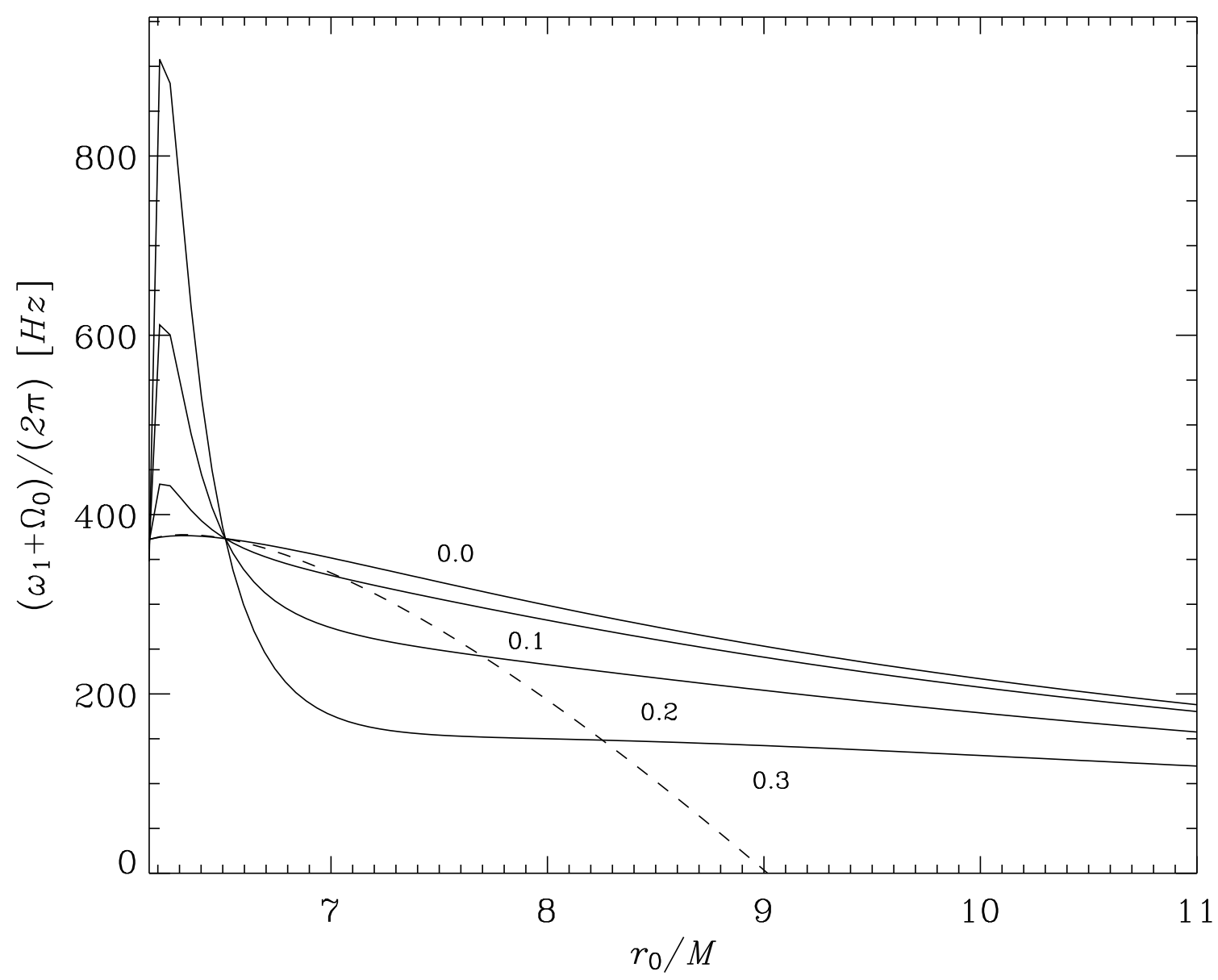

Fig. 6.- The sum of the axisymmetric radial epicyclic mode frequency $\omega_{1}$ and the orbital frequency $\Omega_{0}$ at the pressure maximum, for an $n=3$ pseudo-Newtonian torus around a $M=10 M_{\odot}$ black hole, as a function of $r_{0}$ for different values of $\beta$. Physical tori exist only to the right of and above the dashed line. 


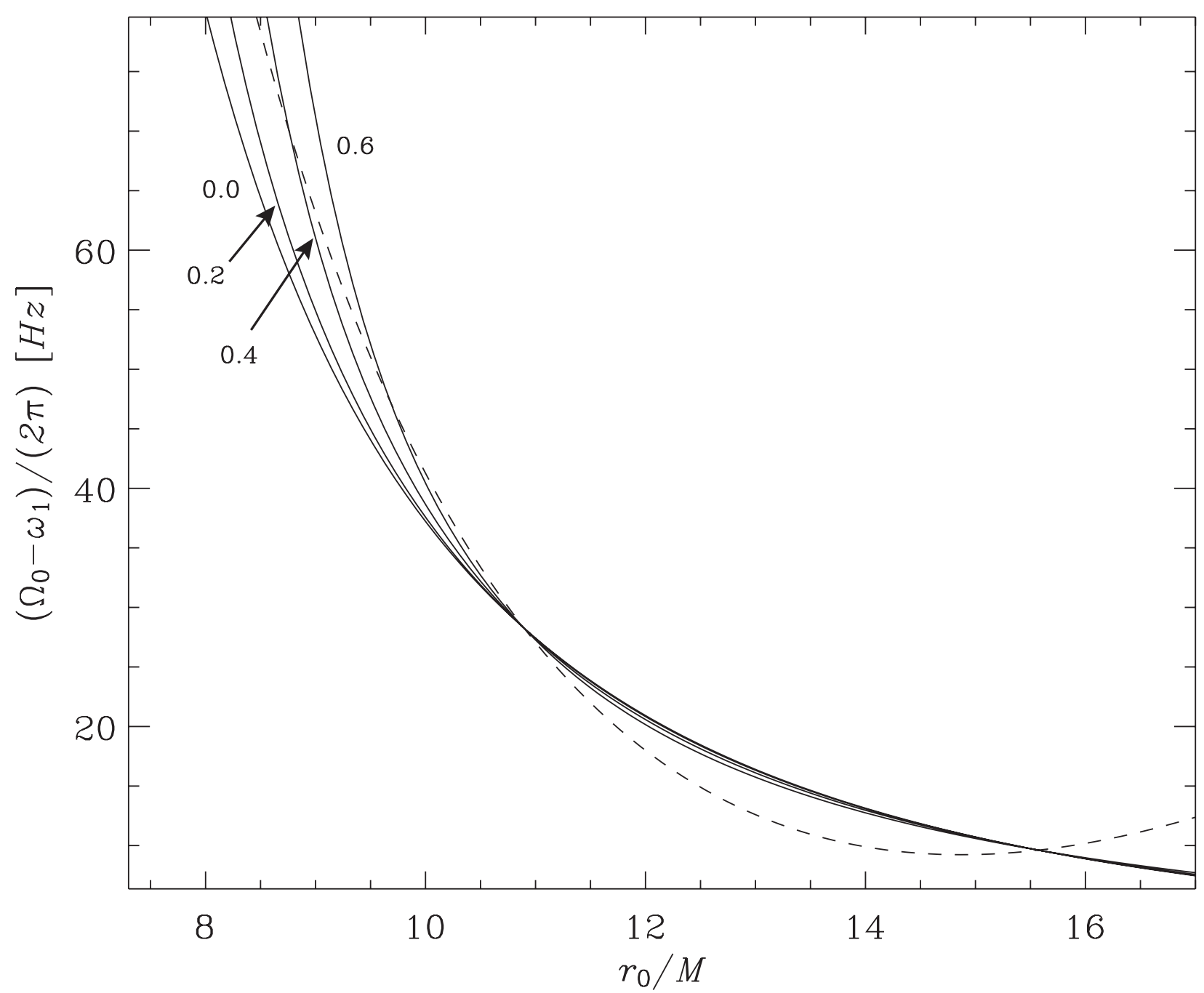

Fig. 7.- The difference between the orbital frequency $\Omega_{0}$ at the pressure maximum $r_{0}$ and the axisymmetric radial epicyclic mode frequency for an $n=3$ pseudo-Newtonian torus around a $M=10 M_{\odot}$ black hole, as a function of $r_{0}$ for different values of $\beta$. Between the radii $r=6 M$ and $r=10.895 M$ the frequency difference increases with increasing $\beta$, and physical tori exist only below the dashed line. From $r=10.985 \mathrm{M}$ to $r=15.573 \mathrm{M}$, the frequency difference decreases slightly with increasing $\beta$, and physical tori exist only above the dashed line. For radii greater than $15.573 M$ the behavior is similar to that of the first interval. 

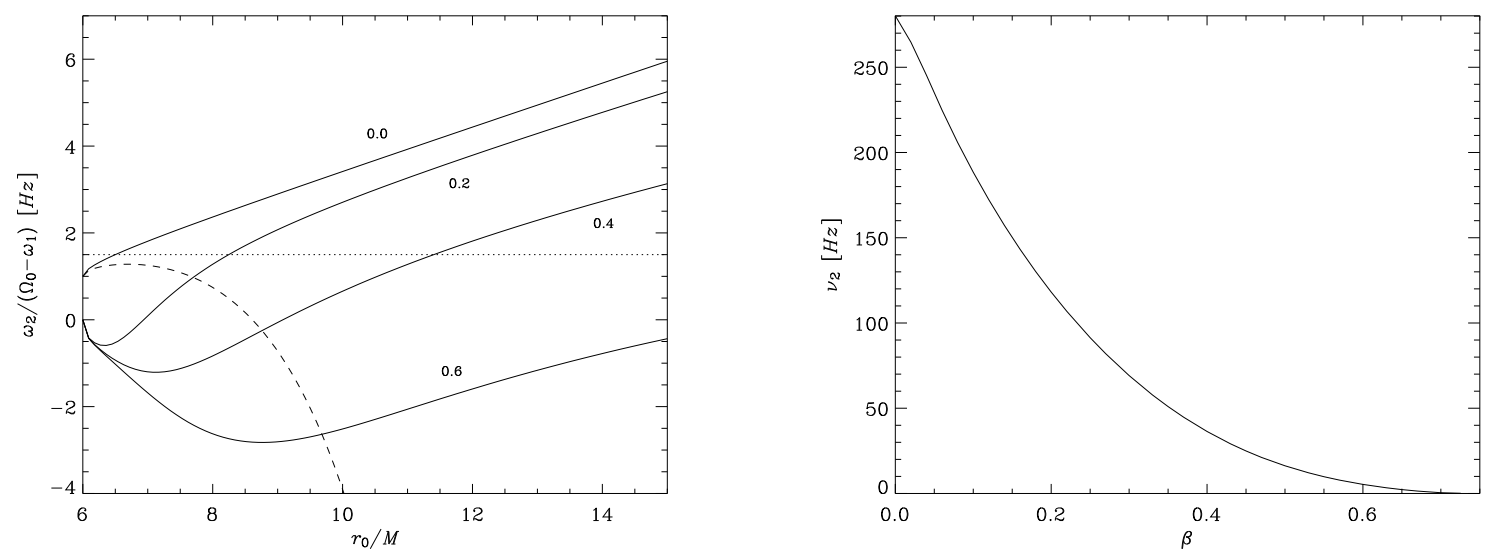

Fig. 8. - Left: The ratio $\omega_{2} /\left(\Omega_{0}-\omega_{1}\right)$ of the axisymmetric vertical mode frequency to the frequency of the $(m=-1)$ radial mode for an $n=3$ torus in a pseudo-Newtonian potential, plotted for different values of $\beta$ as a function of radius of the torus pressure maximum. Physical models lie above the dashed line. For all thick tori having $\beta<\beta_{\max }=0.725108$, there is one possible location of the torus pressure maximum at which the modes are in a $3: 2$ ratio. For tori with $\beta \geq \beta_{\max }$, no such pressure maximum radii can exist. Right: The axisymmetric vertical epicyclic mode frequency $\nu_{2}=\omega_{2} /(2 \pi)$ (for the same tori as in the left panel) at the radius where $\omega_{2} /\left(\Omega_{0}-\omega_{1}\right)=3 / 2$, plotted as a function of $\beta$. At $\beta=\beta_{\text {max }}=0.725108$, the frequency $\nu_{2}$ goes to zero.
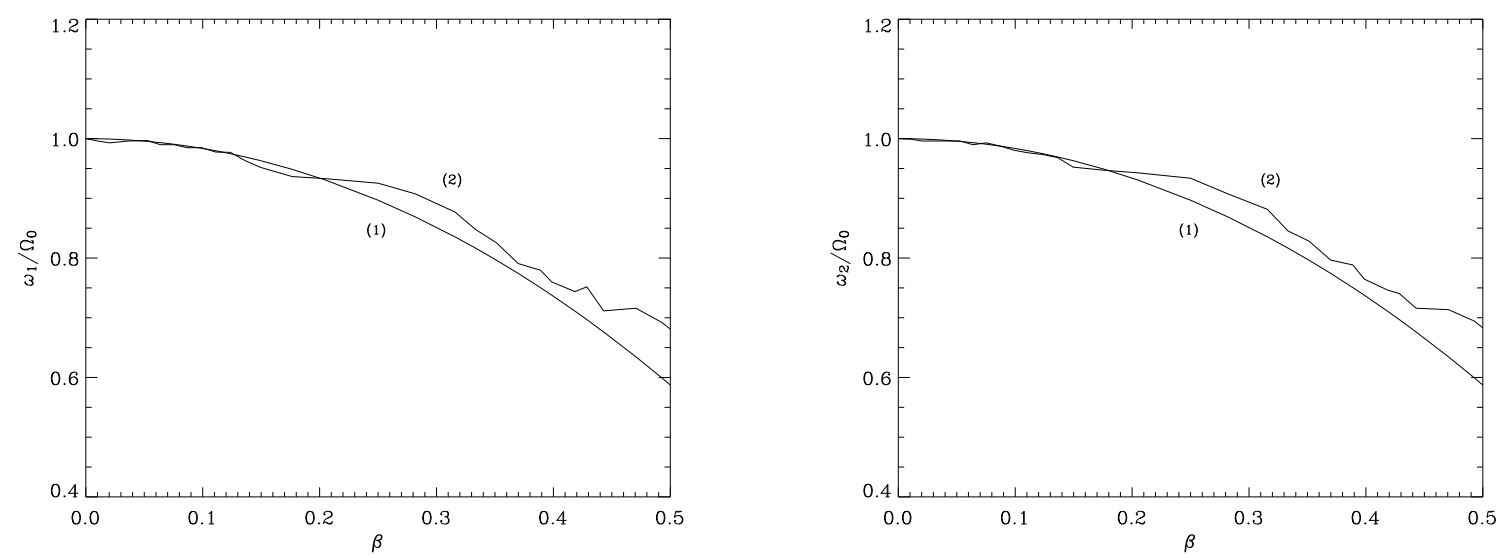

Fig. 9.- Left: Comparison between the analytically (1) and numerically (2) calculated axisymmetric radial epicyclic mode frequency for an $n=3$ torus orbiting in a spherically symmetric point mass potential, plotted as a function of $\beta$. Right: Same as the left panel, but for the axisymmetric vertical epicyclic mode. 

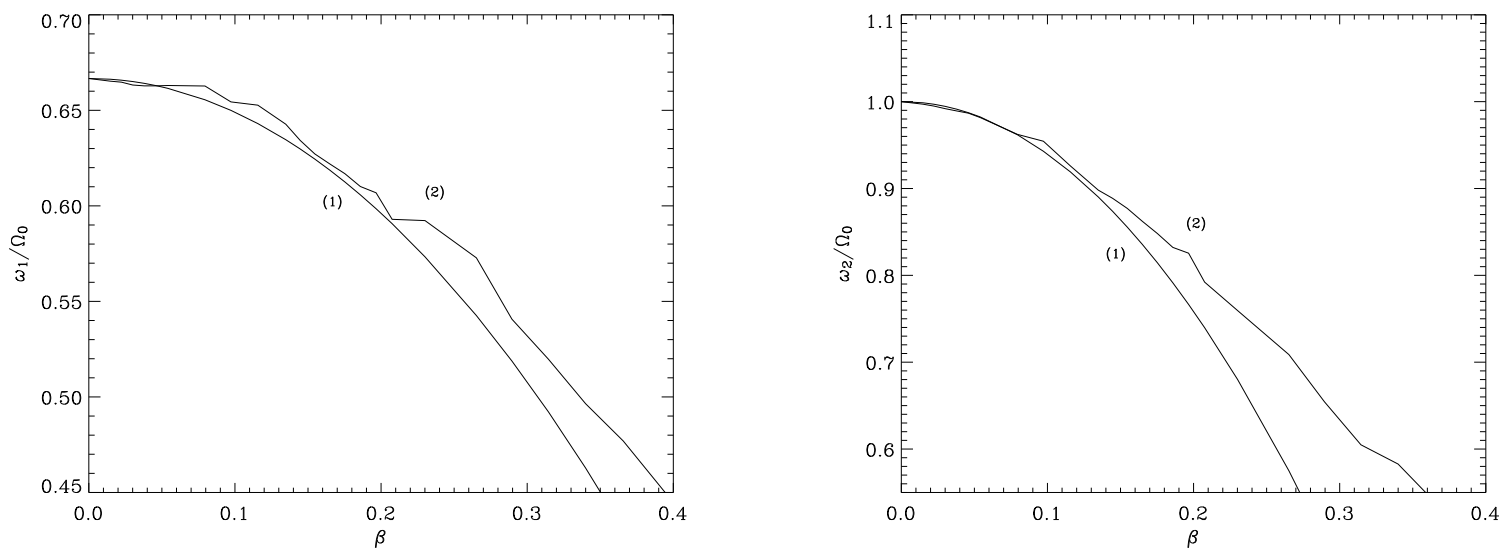

Fig. 10.- Left: Comparison between the analytically (1) and numerically (2) calculated axisymmetric radial epicyclic mode frequency for an $n=3$ torus orbiting in a pseudoNewtonian potential, plotted as a function of $\beta$. The pressure maximum of the torus is at $9.2 M$. For this radius, the maximum value of $\beta$ for which an equilibrium torus can exist, is 0.492968. Right: Same as the left panel, but for the axisymmetric vertical epicylic mode. 
Table 1. Simplest Modes of the General, Constant Specific Angular Momentum Slender Torus.

\begin{tabular}{|c|c|c|}
\hline$J^{\mathrm{a}}$ & $\bar{\sigma}_{0}^{2}$ & Eigenfunction ${ }^{\mathrm{b}}$ \\
\hline 0 & 0 & $a_{0}$ \\
\hline 1 & $\bar{\omega}_{r}^{2}$ & $a_{1} \bar{x}$ \\
\hline 2 & $\bar{\omega}_{z}^{2}$ & $a_{2} \bar{y}$ \\
\hline 3 & $\bar{\omega}_{r}^{2}+\bar{\omega}_{z}^{2}$ & $a_{3} \bar{x} \bar{y}$ \\
\hline 4 & $\frac{(2 n+1)\left(\bar{\omega}_{r}^{2}+\bar{\omega}_{z}^{2}\right)-\left[4 n(n+1)\left(\bar{\omega}_{z}^{2}-\bar{\omega}_{r}^{2}\right)^{2}+\left(\bar{\omega}_{r}^{2}+\bar{\omega}_{z}^{2}\right)^{2}\right]^{1 / 2}}{2 n}$ & $a_{4}\left\{1+\frac{n \bar{\sigma}_{0}^{2}}{\bar{\omega}_{z}^{2}-\bar{\omega}_{r}^{2}}\left(\bar{\omega}_{r}^{2} \bar{x}^{2}-\bar{\omega}_{z}^{2} \bar{y}^{2}\right)-\frac{2(n+1) \bar{\omega}_{z}^{2} \bar{\omega}_{r}^{2}}{\bar{\omega}_{z}^{2}-\bar{\omega}_{r}^{2}}\left(\bar{x}^{2}-\bar{y}^{2}\right)\right\}$ \\
\hline 5 & $\frac{(2 n+1)\left(\bar{\omega}_{r}^{2}+\bar{\omega}_{z}^{2}\right)+\left[4 n(n+1)\left(\bar{\omega}_{z}^{2}-\bar{\omega}_{r}^{2}\right)^{2}+\left(\bar{\omega}_{r}^{2}+\bar{\omega}_{z}^{2}\right)^{2}\right]^{1 / 2}}{2 n}$ & $a_{5}\left\{1+\frac{n \bar{\sigma}_{0}^{2}}{\bar{\omega}_{z}^{2}-\bar{\omega}_{r}^{2}}\left(\bar{\omega}_{r}^{2} \bar{x}^{2}-\bar{\omega}_{z}^{2} \bar{y}^{2}\right)-\frac{2(n+1) \bar{\omega}_{z}^{2} \bar{\omega}_{r}^{2}}{\bar{\omega}_{z}^{2}-\bar{\omega}_{r}^{2}}\left(\bar{x}^{2}-\bar{y}^{2}\right)\right\}$ \\
\hline
\end{tabular}

a $J$ is an arbitrary nonnegative integer index which we are using to label the modes. A more physically descriptive set of labels can be found in the paper by Blaes. Arras \& Fragile (2006).

${ }^{\mathrm{b}}$ The constants $\left\{a_{0}, a_{1}, \ldots, a_{5}\right\}$ are given in Table 2, and are chosen such that the eigenfunctions are normalized in the inner product 34 . 
Table 2. Normalization Constants for the Eigenmodes of Table 1.

\begin{tabular}{cc}
\hline \hline$J$ & $a_{J}$ \\
\hline 0 & $\left(\frac{n \bar{\omega}_{r} \bar{\omega}_{z}}{\pi}\right)^{1 / 2}$ \\
1 & $a_{0}\left[2(n+1) \bar{\omega}_{r}^{2}\right]^{1 / 2}$ \\
2 & $a_{0}\left[2(n+1) \bar{\omega}_{z}^{2}\right]^{1 / 2}$ \\
3 & $a_{0}\left[4(n+1)(n+2) \bar{\omega}_{r}^{2} \bar{\omega}_{z}^{2}\right]^{1 / 2}$ \\
4 & $a_{0}\left\{\frac{(n+2)\left[\bar{\sigma}_{0}^{2}-\left(\bar{\omega}_{z}^{2}+\bar{\omega}_{r}^{2}\right)\right]}{2 n \bar{\sigma}_{0}^{2}-(2 n+1)\left(\bar{\omega}_{z}^{2}+\bar{\omega}_{r}^{2}\right)}\right\}^{1 / 2}$ \\
5 & $a_{0}\left\{\frac{(n+2)\left[\bar{\sigma}_{0}^{2}-\left(\bar{\omega}_{z}^{2}+\bar{\omega}_{r}^{2}\right)\right]}{2 n \bar{\sigma}_{0}^{2}-(2 n+1)\left(\bar{\omega}_{z}^{2}+\bar{\omega}_{r}^{2}\right)}\right\}^{1 / 2}$ \\
\hline
\end{tabular}

